\title{
FROM THE FERMI LIQUID TOWARDS THE WIGNER SOLID IN TWO DIMENSIONS
}

\author{
Jean-Louis Pichard ${ }^{a}$, Giuliano Benenti, Georgios Katomeris \\ Franck Selva and Xavier Waintal \\ Service de Physique de l'Etat Condensé, CEA-Saclay, 91191 Gif sur Yvette cedex, \\ France \\ ${ }^{a}$ Pichard@drecam.saclay.cea.fr
}

To appear in: Exotic States in Quantum Nanostructures ed. by S. Sarkar, Kluwer, Dordrecht.

\begin{abstract}
The quantum-classical crossover from the Fermi liquid towards the Wigner solid is numerically revisited, considering small square lattice models where electrons interact via a Coulomb $U / r$ potential. We review a series of exact numerical results obtained in the presence of weak site disorder for fully polarized electrons (spinless fermions) and when the spin degrees of freedom are included. A novel intermediate regime between the Fermi system of weakly interacting localized particles and the correlated Wigner solid is obtained. A detailed analysis of the non disordered case shows that the intermediate ground state is a solid entangled with an excited liquid. For electrons in two dimensions, this raises the question of the existence of an unnoticed intermediate liquid-solid phase. Using the Coulomb energy to kinetic energy ratio $r_{s} \propto U \propto n_{s}^{-1 / 2}$, we discuss certain analogies between the numerical results obtained as a function of $U$ for a few particles and the low temperature behaviors obtained as a function of the carrier density $n_{s}$ in two dimensional electron gases. Notably, the new "exotic state of matter" numerically observed at low energies in small clusters occurs at the same intermediate ratios $r_{s}$ than the unexpected low temperature metallic behavior characterizing dilute electron gases. The finite size effects in the limit of strong disorder are eventually studied in the last section, providing two numerical evidences that the weak coupling Fermi limit is delimited by a second order quantum phase transition when one increases $U$.
\end{abstract}


Keywords:

- Numerical studies of lattice models with Coulomb repulsions.

- Intermediate liquid-solid quantum phase.

- Metal-insulator transition in two dimensions.

- Finite size scaling with Coulomb repulsions.

"The very simplest form of the theory of the energy bands in metals gave for many problems such accurate explanations of often very intricate properties of metals and alloys that it may well appear superfluous to consider extensions of the simple form of the theory". Those words written by Wigner [1] in 1938 come again as an objection against the need to develop a more rigourous theory, since the Fermi liquid theory (FLT) was improved by Landau [2] in the sense of a perturbation theory based on renormalized single-particle excitations and adapted to include the effects of elastic scattering by the impurities [3]. The need to go outside conventional FLT for explaining the unexpected two dimensional metallic phase [4] discovered by Kravchenko and Pudalov is a subject of controversy. On one hand, certain characteristic FLT behaviors [5] seem to remain in the vicinity of the metal-insulator transition (MIT), suggesting that an "apparent" metallic behavior could be the consequence of "classical" effects (interband scattering [6], temperature dependent screening [7], temperature dependent scattering [8, 9] or classical percolation [10]). On the other hand, the observation of an unexpected MIT is first the result (see for instance Refs. [11, 12]) of new possibilities of studying controlled many body systems which are closer to the strong coupling limit than the previously studied systems in two dimensions. This gave us the motivation to numerically revisit the classic problem of the crossover from the weak coupling Fermi limit towards the strong coupling Wigner limit for electrons in two dimensions. In this chapter, we review our main numerical results. The interest of the information given from exact diagonalization of small systems can be questionned, but it may have the merit to raise questions which may be relevant for explaining the behaviors observed around the "two dimensional MIT".

\section{WEAK AND STRONG COUPLING LIMITS}

A convenient measure of the electron gas density $n_{s}$ is the dimensionless parameter $r_{s}$

$$
r_{s}=\frac{1}{\sqrt{\pi n_{s}} a_{B}^{*}}
$$


defined as the radius $1 / \sqrt{\pi n_{s}}$ of the unit disk divided by the Bohr radius $a_{B}=\hbar^{2} /\left(m e^{2}\right)$. The unit disk encloses an area equal to the area per electron of the gas. For a real two dimensional electron gas (2DEG) created in a field effect device, one uses an effective Bohr radius $a_{B}^{*}=\hbar^{2} \epsilon /\left(m^{*} e^{2}\right)$ which includes the dielectric constant $\epsilon$ of the medium in which the 2DEG is created and the effective mass $m^{*}$ of the carriers. Wigner was the first to consider the dilute limit where $r_{s}$ becomes large, the Coulomb interactions dominate the kinetic energy in determining the wave function and the electrons tend to arrange themselves in a regular lattice. It may be argued that a lattice configuration is not consistent with the translational symmetry characterizing the 2DEG Hamiltonian in the absence of a random substrate. This objection can be removed by forming a new wave function which is a linear combination of all translations of the original lattice: the resultant wave function will have a uniform electronic charge density, as symmetry demands, with an unchanged energy. Originally, Wigner assumed a bcc electron lattice. It was later shown [13] that the hexagonal lattice has a lowest electrostatic energy in two dimensions.

As explained in Refs. $[14,15,16]$, when one considers the Hamiltonian of $N$ electrons in two dimensions,

$$
H=\frac{1}{r_{s}^{2}} \sum_{i}^{N} \nabla_{i}^{2}+\frac{2}{r_{s}} \sum_{\substack{i, j \\ i \neq j}}^{N} \frac{1}{\left|r_{i}-r_{j}\right|}
$$

where the lengths are given in units of $1 / \sqrt{\pi n_{s}}$, the question has been from the early days to obtain the asymptotic behaviors of the ground state energy $E_{0}$ around the weak coupling limit $\left(r_{s}<<1\right)$ :

$$
E_{0}=\frac{h_{0}}{r_{s}^{2}}+\frac{h_{1}}{r_{s}}+0\left(\ln r_{s}\right)
$$

and around the strong coupling limit $\left(r_{s}>>1\right)$ :

$$
E_{0}=\frac{f_{0}}{r_{s}}+\frac{f_{1}}{r_{s}^{3 / 2}}+\frac{f_{2}}{r_{s}^{2}}+0\left(r_{s}^{-5 / 2}\right),
$$

to calculate the coefficients $h$ and $f$, and to discuss the expected range of validity for those asymptotic expansions. Then, one can try to numerically determine the value of $r_{s}$ where the weak coupling energy exceeds the large coupling energy. This can be done at the price of certain approximations which are controlled in the two limits and remain more uncertain in the middle, and after extrapolating finite size studies towards the thermodynamic limit. 
The most advanced works in this field are Quantum Monte Carlo studies $[16,17]$ of systems involving many electrons (typically more than $10^{2}$ ) and assuming two trial wave functions $\Psi_{T}(R)$ adapted to describe either the weak coupling limit, or the strong coupling limit. $\Psi_{T}(R)$ are of the Slater-Jastrow form

$$
\Psi_{T}(R)=D(R) \exp \left(-\sum_{i<j}^{N} u\left(\left|r_{i}-r_{j}\right|\right)\right),
$$

where $D(R)$ is a Slater determinant of extended plane waves for weak coupling, of localized single-particle orbitals for strong coupling. The liquid and crystal pseudopotentials $u(r)$ are repulsive and include in an approximate way the effects of electronic correlations. Then a simple variational approach, or a more involved fixed node Green's-function approach, are used to obtain the energies $E_{0}\left(r_{s}\right)$ dictated by the chosen $\Psi_{T}(R)$ or by its nodal structure. Both the variational energies and the fixed node energies give an upper bound to the exact energy. Comparing for intermediate $r_{s}$ the energies given by the $\Psi_{T}(R)$ adapted to describe the liquid and the crystal, one concludes [17] that there is a first order quantum liquid-solid transition at $r_{s} \approx 37$, with a possible division $[16,18]$ of the liquid phase into a non polarized liquid at small $r_{s}$ and a polarized liquid for larger $r_{s}$. However, the nature of the quantum mechanism of melting is still debated, and the possibility of a continuous transition has been very recently proposed[19]. The solid is assumed to be a frustrated antiferromagnet[20] before becoming ferromagnetic [21] at very large $r_{s}$. The same Monte Carlo method has been used[22] in the presence of impurities. The conclusion was that disorder can stabilize the solid to weaker values of $r_{s}$.

Andreev and Lifshitz have discussed [23] in 1969 the possibility to have a more complex intermediate state between the solid and the liquid, which should be neither a solid nor a liquid. Two kinds of motion should be possible in it; one possesses the properties of motion in an elastic solid, the second possesses the properties of motion in a liquid. This idea comes from a theory of defects in quantum solids. The nature of the relevant defects is a complicated issue. Let us give a possible example: a vacancy yielded by one electron hopping from the Wigner lattice towards some interstitial site. In a classical solid, this defect has a certain electrostatic cost and remains localized. In a quantum solid, we have in addition the tunneling effect, and if this defect can be created, it will be delocalized since the system is invariant under translation. Therefore, when $r_{s}$ decreases from the strong coupling limit, the increasing band width of the zero point defects of this type may exceed their decreasing electrostatic energy cost, leading to two possibilities for 
intermediate $r_{s}$ : Either the total melting of the solid to directly give a liquid, as implicitely assumed for instance in Refs. [19], or a quantum floppy solid coexisting with a liquid of delocalized defects, as conjectured by Andreev and Lifshitz. A phenomenological FLT theory à la Landau of such gapless "delocalized excitations" of a floppy quantum solid has been later proposed in Ref. [24]. The discussion of this second possibility is one of the central points of this chapter.

\section{DILUTE 2DEG IN FIELD EFFECT DEVICES}

A pure 2DEG can be realized by trapping electrons on the surface of liquid helium, but it is difficult to reach a sufficient density to study the quantum regime. Another possible realization is given by the new classes of superconducting cuprates where the electronic motion is essentially two dimensional. The charge density can be varied by chemical doping, and a complex phase diagram is obtained, with insulating, superconducting and metallic behaviors.

Eventually, one can create a two dimensional gas of charges (electrons or holes) at the interface between two doped semiconductors (GaAsAlGaAs heterostructures), between a semi-conductor and an insulator (Si-Mosfet), or very recently [12] between an organic crystal (pentacene, tetracene and anthracene) and an insulator. The carrier density can be varied by a gate from a very dilute limit towards larger densities. In Fig. 1, we mention the remarkable phenomena observed in organic (left) or doped semi-conductor (right) field effect devices. A clean interface may give a high carrier mobility, may allow the observation [26] of the fractional quantized Hall effect (FQHE) and may give a measurable conductivity in a very dilute limit (typically $n_{s} \approx 10^{9}-10^{11}$ carriers per $\mathrm{cm}^{2}$ ) for GaAs heterostructures and organic devices. If the effective mass of the carriers is large enough (a condition which is not satisfied by the electrons in Ga-As heterostructures) the effective factor $r_{s}$ can be in the vicinity of the values where the Fermi-Wigner crossover is expected. One of the surprises in those high quality field effect transistors has been the observation of a metallic low temperature behavior $[4,12,27]$ in a certain intermediate range of carrier densities, where a large perpendicular magnetic field yields FQHE or magnetically induced Wigner crystals [28].

\section{METAL-INSULATOR TRANSITION}

A recent review of the 2D-MIT can be found in Ref. [4] with an extended list of references. We summarize by a few sketches some of the 


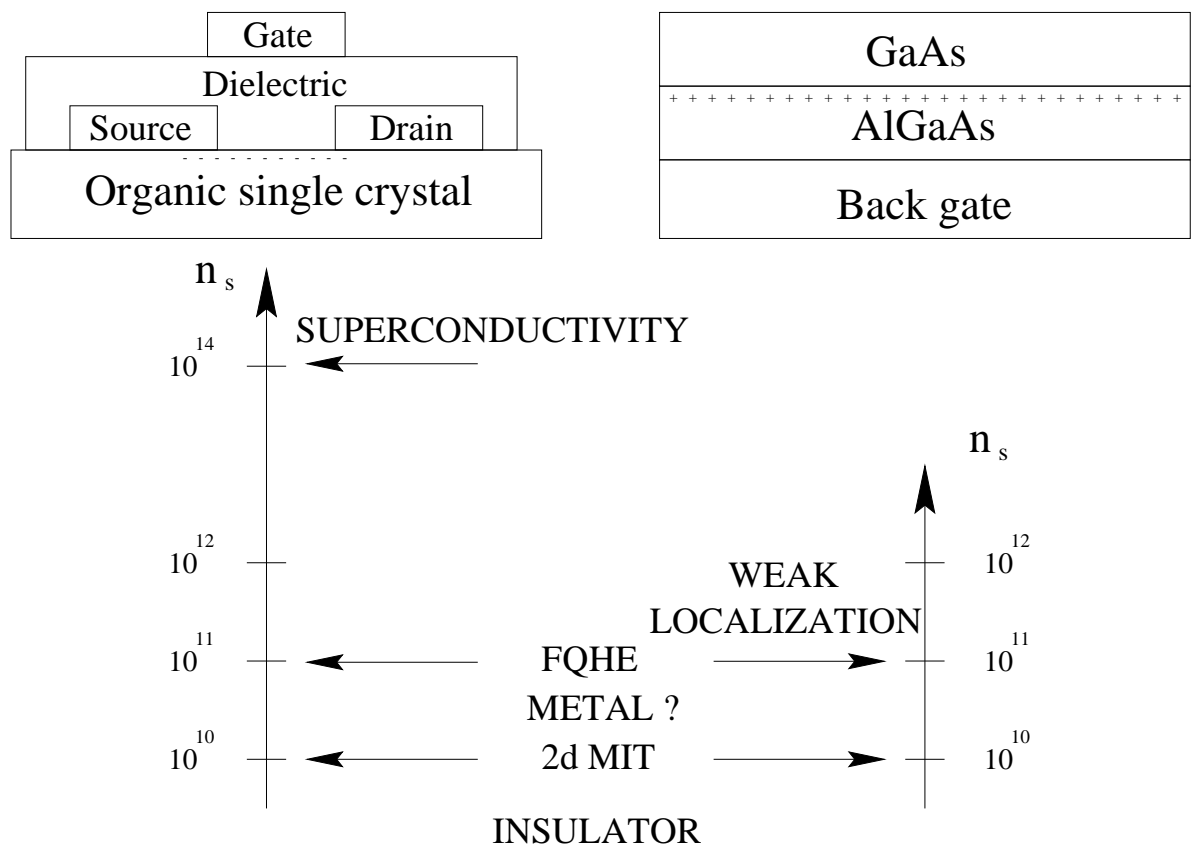

Figure 1. Schematic picture of an organic field effect transistor (upper left) and of a GaAs heterostructure (upper right) where the location of the 2DEG or 2DHG (symbol plus for the holes) is indicated. The characteristic low temperature behaviors are summarized below as a function of the (typical) carrier density $n_{s}\left(\mathrm{~cm}^{-2}\right): 2 \mathrm{dMIT}$, $2 d$ metal, FQHE for a sufficient magnetic field, weak localization correction to the Boltzmann conductivity, superconductivity (see Ref.[25]). The densities give a typical order of magnitude, the observed behaviors depending also on the effective mass of the carriers.

behaviors which have been observed, and which are useful for discussing our numerical results. The main surprise was given by the temperature dependence of the 2DEG resistivity $\rho(T)$ around a low critical density $n_{c 1}$. As sketched in Fig. 1 (see Fig.1 of Ref. [29]), $\rho(T)$ decreases as a function of $T$ when $n_{s}<n_{c 1}$, becomes temperature independent at $n_{s}=n_{c 1}$ and increases when $n_{s}>n_{c 1}$. A decay is the expected behavior for an insulator, while an increase usually characterizes a metal. These behaviors occur [30] in a low temperature range $35 m k<T \leq T_{F}$, where $T_{F} \approx 0.8-5 K$ are the typical Fermi temperatures of those dilute 2DEGs. The temperature increase of $\rho(T)$ can be large for a 2DEG created in a Si-Mosfet (typically one order of magnitude), but remains weak in a 2 DHG created in a GaAs heterostructure. For the densities $n_{s}$ where $\rho(T)$ has a metallic behavior, a parallel field $B$ induces a large positive 


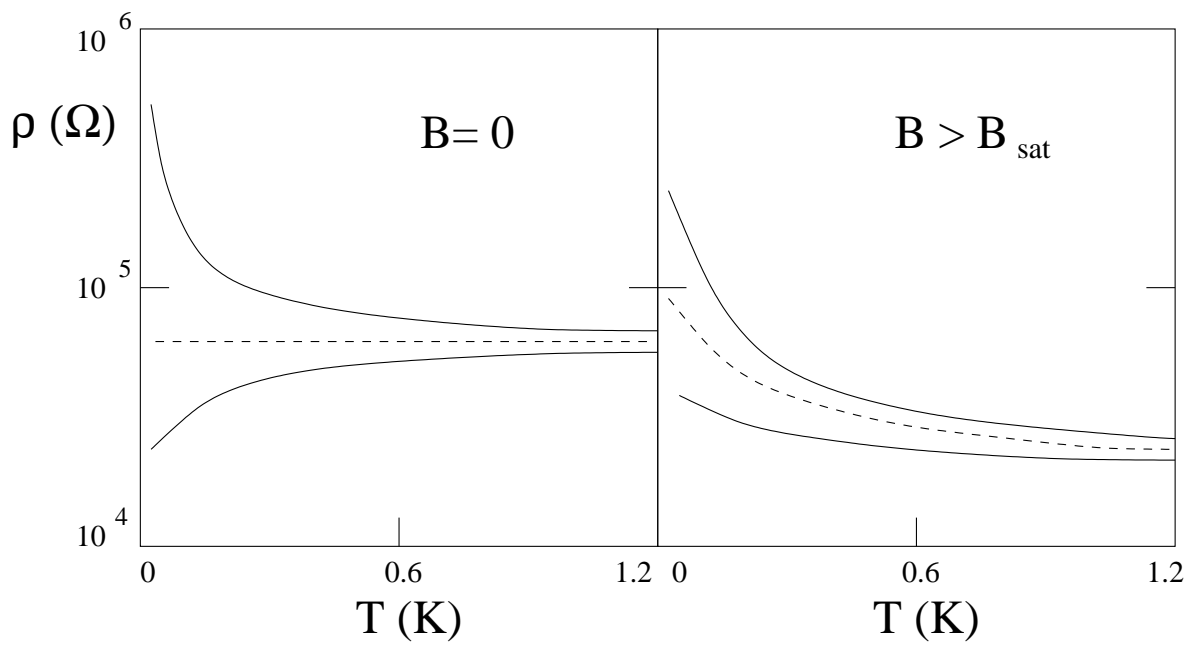

Figure 2. Resistivity versus temperature without (left) and with (right) a large parallel magnetic field $B>B_{\text {sat }} . n_{s}<n_{c 1}$ (upper curves), $n=n_{c 1}$ (middle curves) and $n>n_{c 1}$ (lower curves).

magnetoresistance which saturates above a certain field $B_{\text {sat }}$, as sketched in Fig.3 (see Fig. 1 of Ref. [31] and Fig. 3 of Ref. [32]). From small angle Shubnikov-de Haas measurements done in a Si-mosfet, it was concluded in Ref. [31] that $B_{\text {sat }}$ signals also the onset of full spin polarization. Close to the MIT, $B_{\text {sat }}$ is very small and increases as $n_{s}-n_{c 1}$ above $n_{c 1}$ [32]. This corresponds to the intermediate values of $r_{s}$ (typically $\left.3<r_{s}<10\right)$ where the metallic behavior is observed. When $B>B_{\text {sat }}$, the metallic increase of $\rho(T)$ disappears, but the $I-V$ characteristics sketched in Fig. 4 (Fig 2 of Ref. [29]) indicates the existence of a critical density $n_{c 2}$ below which a non linearity is observed and above which it disappears. The density $n_{c 1}$ and $n_{c 2}$ are close to each others, if not identical when $B=0$. The dependence of the characteristic $n_{c 2}$ as a function of a parallel magnetic field $B$ is sketched in Fig. 4. (Fig. 4 of Ref. [29]).

The critical density $n_{c 1}$ does not give a unique critical value for the factor $r_{s}$. Impurity scattering plays a role. For clean systems, one needs to have a much larger factor $r_{s}$ than in a dirty system, as sketched in Fig. 5. (see inset of Fig. 1 in Ref. [33]). In an (undoped) organic field effect transistor, the 2DEG is less scattered by impurities and the MIT is seen[27] at an even smaller density $\left(r_{s} \approx 50\right)$.

The surprise caused by this unexpected metallic behavior was mainly due to its discrepancy with the scaling theory of localization, which does 

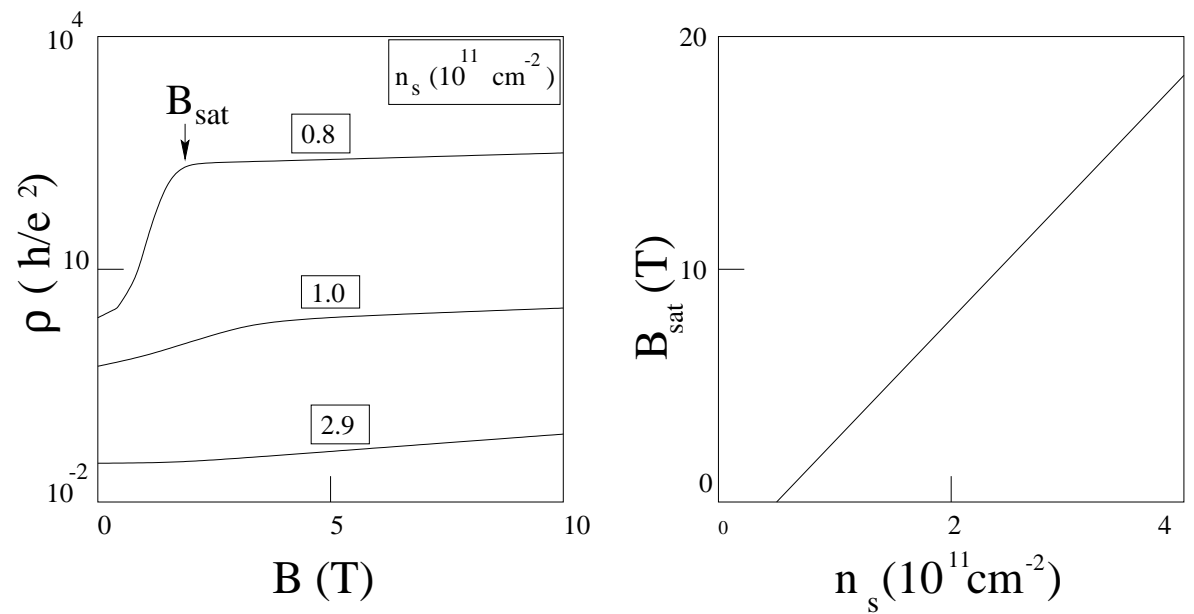

Figure 3. Resistivity versus parallel magnetic field (left) and saturation field $B_{\text {sat }}$ as a function of the carrier density (right).
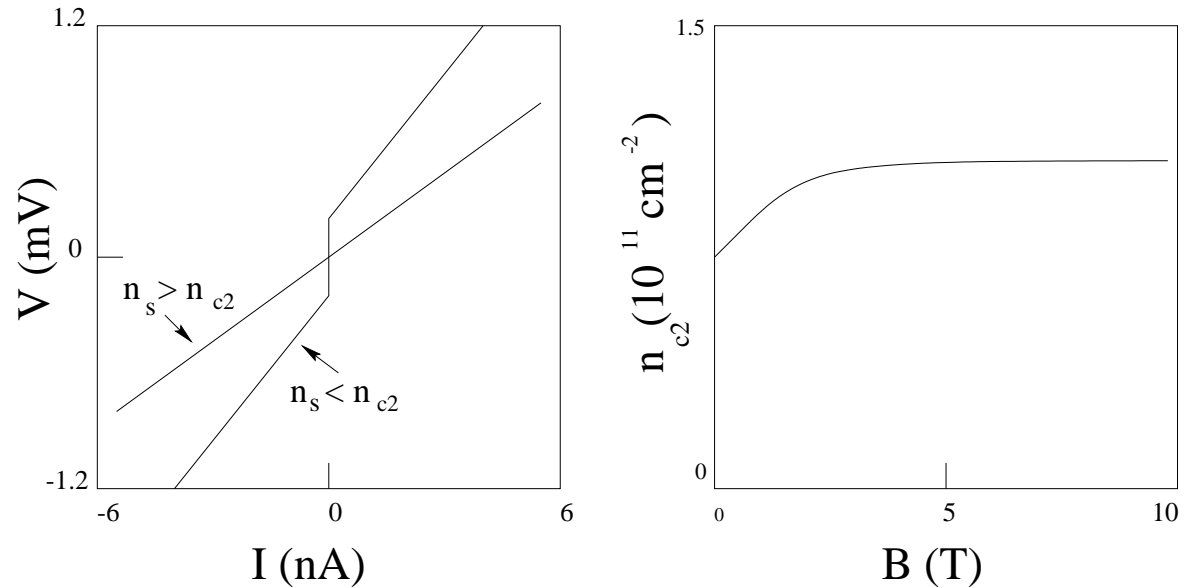

Figure 4. Current-voltage non linear characteristics of the insulating phase which are suppressed above the MIT (left). Critical density $n_{c 2}$ above which the non linearity of the I-V characteristics disappears as a function of the parallel magnetic field.

not take into account electron-electron interactions. When the conductance $g$ is larger than the conductance quantum $e^{2} / h$, a weak disorder perturbative expansion gives for the average conductance a universal logaritmic correction to the Drude conductivity which defavors transport, unless there is a sufficient spin orbit scattering. In the weak disorder 


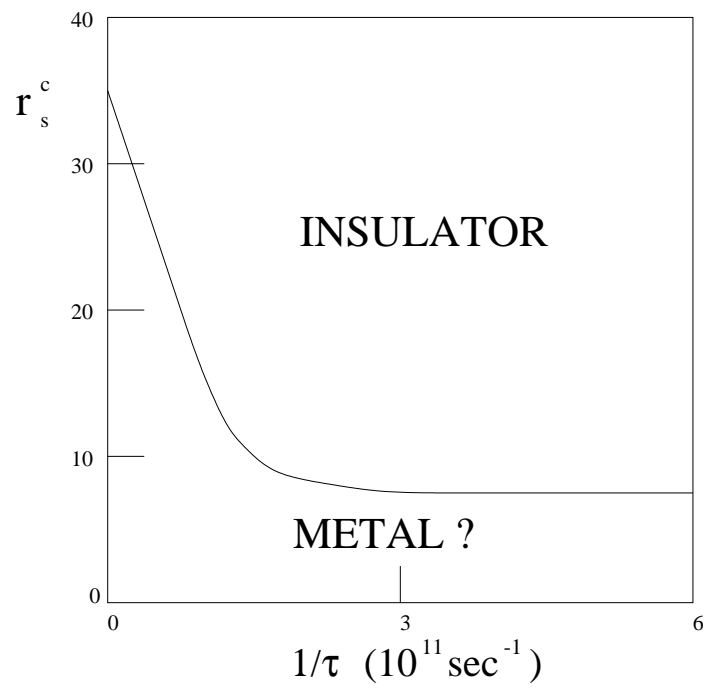

Figure 5. Critical factor $r_{s}^{c}$ at which the MIT is observed as a function of the inverse elastic scattering time.

limit, one can also take into account the interaction when $r_{s}<1$ and one obtains additional corrections which reduce transport in a similar way. However, the extrapolation of the small $r_{s}$ interaction dependent correction to larger $r_{s}$ suggests a possible change of the sign of the corrections due to the interactions, indicating the possibility of a metallic phase in two dimensions, as mentioned by Finkelshtein [34]. Computer calculations without interaction and transport measurements at not too low densities made in the eighties have confirmed the absence of metallic behavior in two dimensions. The difference between the recent experiments giving a MIT and the former experiments confirming the absence of metallic behavior seems to be the quality of the interfaces at which the 2DEG is created. This feature makes possible to have a measurable conductivity at much lower carrier densities than previously. An intermediate range of density, where the factor $r_{s}$ is too large (too small) to allow expansion in powers of $r_{s}\left(1 / r_{s}\right)$ and a weak elastic scattering seems to be necessary for observing the metallic behavior. This hypothesis was supported by Ref. [35] where a study of a 2DHG in a Ga-As heterostructure gives a range $r_{s}^{F}<r_{s}<r_{s}^{W}$ for having a weak metallic behavior in a disordered sample. When $r_{s}<r_{s}^{F}$, one would have weakly interacting quasi-particles dominated by Anderson localization when the temperature $T \rightarrow 0$. When $r_{s}>r_{s}^{W}$, one would have a highly correlated set of charges. Between $r_{s}^{F} \approx 6$ and $r_{s}^{W} \approx 9$ in the studied sample, 
a problematic small metallic behavior is observed between two insulating behaviors of different nature (Anderson insulator for large densities, pinned Wigner solid for low densities). The re-entrant MIT at low $r_{s}$ is not easy to observe, if it exists, since the localization length of a clean device can be very large, and the observation of a possible re-entrant insulating behavior at high densities can require very low temperatures. This is why many observations of a MIT have been reported for $r_{s} \approx 10$, while very few experiments give a possible re-entrant MIT at $r_{s} \approx 3$. Moreover, more recent works [5, 7, 9]) put doubts about the reality of this intermediate metallic behavior when the temperature goes to zero, since the effect of a weak perpendicular magnetic field can be described by usual weak localization theories, even for values of $r_{s}$ as large as 15 (see Fig. 6 taken from Ref. [5]). The hypothesis of a certain temperature dependent screening was suggested for explaining the anomalous temperature dependence, and it was proposed that usual quantum interferences should drive at possibly very low temperatures the system to the formerly expected insulating behavior. Recent measures performed down to $5 m K$ do not confirm[36] this hypothesis. The estimate of the phase breaking length $L_{\phi}$ which is traditionally done for estimating the low temperature dependence of the resistance from a zero temperature theory leads again to the famous problem of the saturation [37] of $L_{\phi}$ when $T \rightarrow 0$, problem leading also to many possible and controversial explanations.

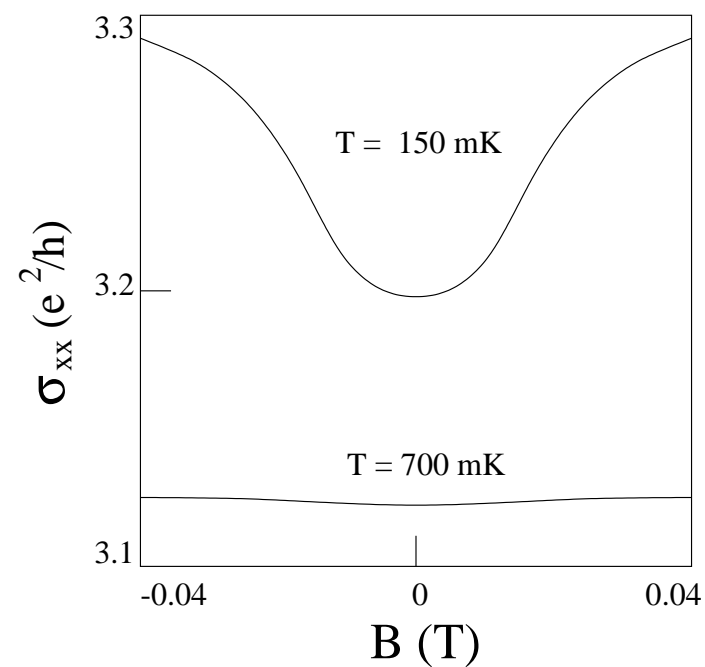

Figure 6. Usual weak localization behavior of the conductance induced by a perpendicular magnetic field in a 2DHG created in Ga-As heterostructure. 
In summary, a significant metallic behavior can be seen using a 2DEG created in a Si-mosfet, while a weaker one occurs in a 2DHG created in a Si-Ge quantum well or a Ga-As hetrostructure. Nevertheless, in the latter system, the study of the compressibility gives complementary signatures $[38,39,40]$ of a possible quantum phase transition. Local compressibility measurements show that the system is more homogenous in the intermediate metallic phase than in the low density insulating phase. Very recently, the possibility that the MIT would be accompanied by a magnetic transition has been suggested [32, 41].

\section{LATTICE MODEL}

The previous experimental observations lead us to numerically revisit the Fermi-Wigner crossover using a two dimensional model describing $N$ particles on $L \times L$ square lattice with periodic boundary conditions (BCs), i.e. with a torus topology. The most general Hamiltonian $\mathcal{H}$ of the lattice model we will focus on reads,

$$
\begin{aligned}
\mathcal{H}= & \sum_{i, \sigma}\left(-t \sum_{i^{\prime}} c_{i^{\prime}, \sigma}^{\dagger} c_{i, \sigma}+v_{i} n_{i, \sigma}\right) \\
& +\frac{U}{2} \sum_{\substack{i, i^{\prime} \\
i \neq i^{\prime}}} \frac{n_{i, \sigma} n_{i^{\prime}, \sigma^{\prime}}}{\left|i-i^{\prime}\right|}+2 U \sum_{i} n_{i, \uparrow} n_{i, \downarrow},
\end{aligned}
$$

where the operators $c_{i, \sigma}\left(c_{i, \sigma}^{\dagger}\right)$ destroy (create) an electron of spin $\sigma$ at the site $i$ and $n_{i, \sigma}=c_{i, \sigma}^{\dagger} c_{i, \sigma}$. $\mathcal{H}$ consists of

- a hopping term $-t$ that couples nearest-neighbor sites, and accounts for the quantum kinetic energy,

- the pairwise electron-electron interaction, which itself consists of a $2 U$ Hubbard repulsion when two electrons are at the same site $i$ with opposite spins and a $U /\left|i-i^{\prime}\right|$ spin independent Coulomb repulsion when they are separated by a distance $\left|i-i^{\prime}\right|$ (smallest distance between the sites $i$ and $i^{\prime}$ on a square lattice with periodic BCs),

- on site random potentials $v_{i}$ which are uniformly distributed inside the interval $[-W / 2, W / 2]$.

The clean system is obtained when the disorder strength $W$ is set to zero. In our model with a lattice spacing $a, \hbar^{2} /\left(2 m^{*} a^{2}\right) \rightarrow t, e^{2} /(\epsilon a) \rightarrow U$, such that the factor $r_{s}$ becomes:

$$
r_{s}=\frac{1}{\sqrt{\pi n_{s}} a_{B}^{*}}=\frac{U}{2 t \sqrt{\pi n_{e}}}
$$




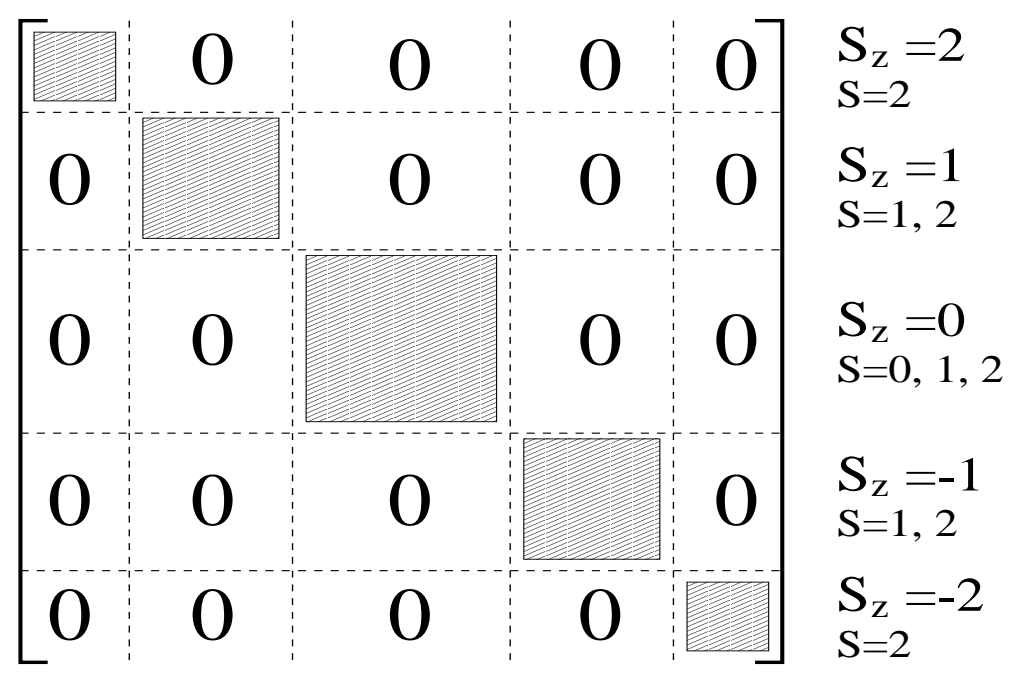

Figure 7. Structure of the Hamiltonian matrix for 4 electrons in a $6 \times 6$ square lattice. The size of the different diagonal non zero sub-blocks are $N\left(S_{z}\right)=396900,257040$ and 58905 for $S_{z}=0, \pm 1, \pm 2$ respectively.

for a filling factor $n_{e}=N / L^{2}$. This dimensionless ratio $r_{s}$ will allow us to compare our results obtained as a function of $U$ for a fixed filling factor $n_{e}$ and the experimental results obtained as a function of $n_{s}$.

We denote $\mathcal{S}$ and $\mathcal{S}_{z}$ the total spin and its component along an arbitrary direction $z$. Since $\left[\mathcal{S}^{2}, \mathcal{H}\right]=\left[\mathcal{S}_{z}, \mathcal{H}\right]=0, \mathcal{H}$ can be written in a block-diagonal form, with $N+1$ blocks where $S_{z}=-N / 2, \ldots, N / 2$ respectively. When $B=0$, there is no preferential direction and the groundstate energy $E_{0}$ does not depend on $S_{z}$. For a groundstate of total spin $S, \mathcal{H}$ has $2 S+1$ blocks with the same lowest eigenenergy $E_{0}\left(S^{2}\right)$ since $E_{0}\left(S^{2}\right)=E_{0}\left(S^{2}, S_{z}\right) ; S_{z}=-S,-S+1, \ldots, S-1, S$. Therefore, the number $N_{b}$ of blocks of different $S_{z}$ and of same lowest energy gives the total spin $S=\left(N_{b}-1\right) / 2$ of the groundstate.

If $N$ and $L$ are small enough, the ground state and the first excitations can be exactly calculated using Lanczos algorithm. Otherwise, certain approximations are unavoidable. Let us focus on the case $N=4$ and $L=6$ where the structure of $\mathcal{H}$ is given in Fig. 7 for different $S_{z}$. Without magnetic field, we have the symmetry $\pm S_{z}$, and we have only to diagonalize the three sub-blocks with $S_{z} \geq 0$. $\mathcal{H}\left(S_{z}=2\right)$ corresponds to fully polarized electrons (spinless fermions) where the orbital part of the wave-functions is totally anti-symmetric. 


\section{STUDIED QUANTITIES}

From exact diagonalization for small systems and using approximations for larger systems and weak coupling, we will study:

- the lowest eigenenergies $E_{n}\left(S_{z}\right)$ of the $S_{z}$ sub-blocks and the corresponding eigenvectors $\mid \Psi_{n}\left(S_{z}\right)>$. $n=0,1,2, \ldots$ corresponds to the states ordered by increasing energies.

- The local persistent currents $\vec{J}(i)$ created at a site $i$ by an Ahronov Bohm flux $\phi$ which is enclosed along the longitunal $l$-direction as sketched in Fig. 8. The flux $\phi$ can be included by taking

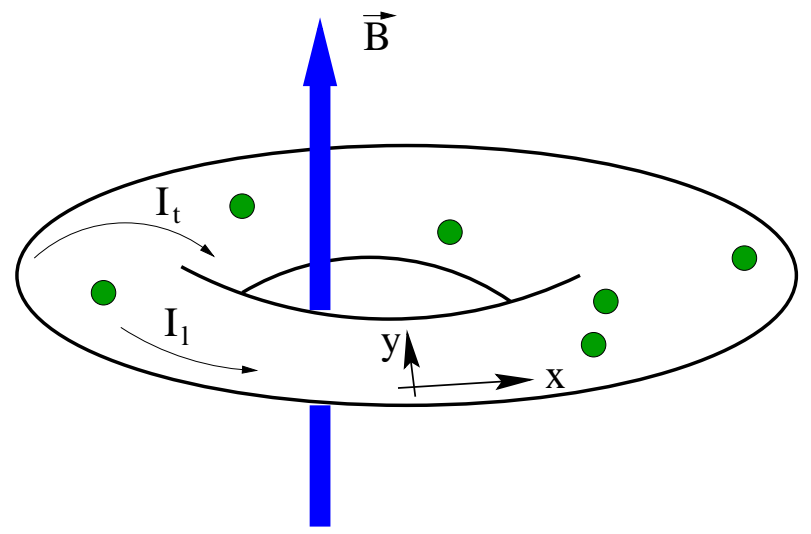

Figure 8. 2D Torus with $N$ electrons enclosing an Aharonov-Bohm flux $\phi=B L_{x}^{2}$

appropriate longitudinal BCs (antiperiodic BCs corresponding to $\phi=\pi$ in our convention). The BCs along the transverse $t$-direction remain periodic. The $\vec{J}(i)$ are vectors defined by their longitudinal and transverse components $\left(\vec{J}(i)=\left(J_{i, l}, J_{i, t}\right)\right)$, or by their angles $\theta_{i}=\arctan \left(J_{i, t} / J_{i, l}\right)$ and their absolute values $J_{i}=\left|\vec{J}_{i}\right|$. The longitudinal component $J_{i, l}$ of an eigenstate $|\Psi\rangle$ is defined as

$$
J_{i, l}=2 \operatorname{Im}\left\langle\Psi\left|c_{i_{x}+1, i_{y}}^{\dagger} c_{i_{x}, i_{y}} \exp (i \phi / L)\right| \Psi\right\rangle
$$

and $J_{i, t}$ is given by

$$
J_{i, t}=2 \operatorname{Im}\left\langle\Psi\left|c_{i_{x}, i_{y}+1}^{\dagger} c_{i_{x}, i_{y}}\right| \Psi\right\rangle
$$


The total current $I^{(n)}$ of the $n^{\text {th }}$ many-body wavefunction $\left|\Psi_{n}\right\rangle$ of energy $E_{n}$ has a total longitudinal component $I_{l}(n)$ given by

$$
I_{l}(n)(\bar{\phi})=-\left.\frac{\partial E_{n}}{\partial \phi}\right|_{\phi=\bar{\phi}}=\frac{\sum_{i} J_{i, l}(n)}{L} .
$$

which will be calculated for $\bar{\phi}=\pi / 2$.

- The crystallization parameter $\gamma$ defined using the function $C(r)=$ $N^{-1} \sum_{i} \rho_{i} \rho_{i-r}$, where $\rho_{i}=\left\langle\Psi\left|n_{i}\right| \Psi\right\rangle$ is the electronic density of the state $|\Psi\rangle$ at the site $i$. The crystallization parameter $\gamma$ is given by

$$
\gamma=\max _{r} C(r)-\min _{r} C(r)
$$

Note that $\gamma=1$ when the $N$ particles are localized on $N$ lattice sites and form a rigid solid and 0 when they are extended on the $L^{2}$ sites and form an homogenous liquid.

- The participation ratio $\chi=N^{2}\left(\sum_{i} \rho_{i}^{2}\right)^{-1}$, which gives the typical number of lattice sites occupied by an eigenstate $|\Psi\rangle$.

- The spectral parameter $\eta$ which characterizes the level repulsion. Uncorrelated spectra exhibit Poisson statistics. Correlated spectra can be described by Random Matrix Theory with WignerDyson (W-D) statistics. For the one body spectra, the distribution $P(s)$ of the normalized energy spacings between consecutive levels has two different forms when $L \rightarrow \infty$ : the Poisson distribution $P_{P}(s)=\exp (-s)$ if the wavefunctions are localized, the Wigner surmises $P_{W}^{O}(s)=(\pi s / 2) \exp \left(-\pi s^{2} / 4\right)$ with time reversal symmetry (TRS) and $P_{W}^{U}(s)=\left(32 s^{2} / \pi^{2}\right) \exp \left(-4 s^{2} / \pi\right)$ without TRS, if the wave functions are extended. For the normalized $N$-body energy spacings $s_{n}=\left(E_{n+1}-E_{n}\right) /<E_{n+1}-E_{n}>$ (the brackets denote ensemble average), we define a spectral parameter:

$$
\eta_{(O, U)}=\frac{\operatorname{var}(P(s))-\operatorname{var}\left(P_{W}^{(O, U)}(s)\right)}{\operatorname{var}\left(P_{P}(s)\right)-\operatorname{var}\left(P_{W}^{(O, U)}(s)\right)},
$$

( $\eta_{O}$ with TRS, $\eta_{U}$ in the absence of TRS, for instance when $\phi=$ $\pi / 2))$. $\operatorname{var}(P(s))$ denotes the variance of $P(s)$. The spectral parameter $\eta=1$ when $P(s)=P_{P}(s)$ and $\eta_{(O, U)}=0$ when $P(s)=$ $P_{W}^{(O, U)}(s)$.

- The Zeeman energy necessary to polarize a non magnetized cluster. A parallel magnetic field $B$ does not induce orbital or AharonovBohm effects, but defines the $z$-direction and removes the $S_{z}$ degeneracy by the Zeeman energy $-g \mu B S_{z}$. The ground state energy 
and its magnetization are given by the minimum of $E_{0}\left(S^{2}, S_{z}, B=\right.$ $0)-g \mu B S_{z}$. For a $S=0$ groundstate without field, the value $B^{*}$ for which $E_{0}\left(S_{z}\right)-g \mu B^{*} S_{z}=E_{0}\left(S_{z}=0\right)$ defines the field necessary to polarize the system to $S \geq S_{z}$. If one studies $N=4$ electrons, the total $Q_{2}=E_{0}\left(S_{z}=2\right)-E_{0}\left(S_{z}=0\right)$ and partial $Q_{1}=E_{0}\left(S_{z}=1\right)-E_{0}\left(S_{z}=0\right)$ polarization energies give the Zeeman energies necessary to yield $S=2$ and $S=1$ respectively for a cluster with $S=0$.

\section{INTERMEDIATE COUPLING REGIME FOR SPINLESS FERMIONS AND WEAK DISORDER}

We first consider an ensemble of disordered clusters with $L=6$ and $N=4$. The ground state (GS) and the first excitations of the fully polarized sub-block ( $S_{z}=2$, spinless fermions) of the Hamiltonian matrix shown in Fig.7 have been obtained using the Lanczos algorithm. The statistical ensemble typically includes $10^{3}-10^{4}$ samples obtained from a disorder distribution with $W=5$. This is a relatively weak disorder for which one has quantum diffusion when $r_{s}=0$ inside the small clusters (no Anderson localization).

\subsection{GROUND STATE}

We summarize in this subsection the main results published in Ref. [42] and complementary unpublished results. As one switches on $U$, a first characteristic threshold $r_{s}^{F}$ can be identified by looking at the average total longitudinal persistent current $I_{l}$ of the GS at $\phi=\pi / 2$, and comparing the exact quantity with the Hartree-Fock (HF) approximation (see appendix). Below $r_{s}^{F} \approx 5$, the mean field approximation reproduces the exact $I_{l}$, but strongly underestimates $I_{l}$ above $r_{s}^{F}$. This sharp breakdown of the HF approximation shown in Fig. 9 means that strong correlation effects occur above $r_{s}^{F}$, such that the shift the GS energy when the BCs are changed cannot be obtained assuming the best possible SD for the ground state.

A closer investigation of the persistent currents on a typical sample gives three regimes, as shown in Fig.10. See also Ref. [43]. For weak coupling, the local currents flow randomly inside the cluster, due to elastic scattering on the site potentials. For intermediate coupling, the pattern of the persistent currents becomes oriented along the shortest direction enclosing $\phi$. For large coupling, the oriented currents vanish. Ref. [44] gives a detailed study of the large coupling limit where one can use perturbation theory for having the sign and the magnitude of $I_{l}$. 


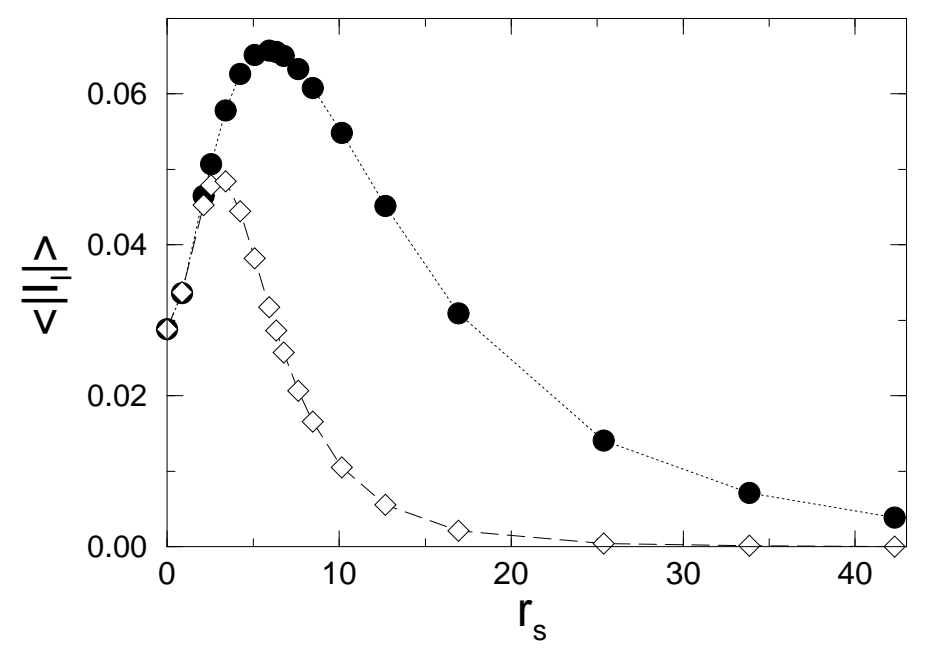

Figure 9. Ensemble average longitudinal GS current $\left\langle I_{l}\right\rangle$ as a function of $r_{s}$ for $N=4, L=6$ and $W=5$. Exact values (filled symbols) and HF values (empty symbols).
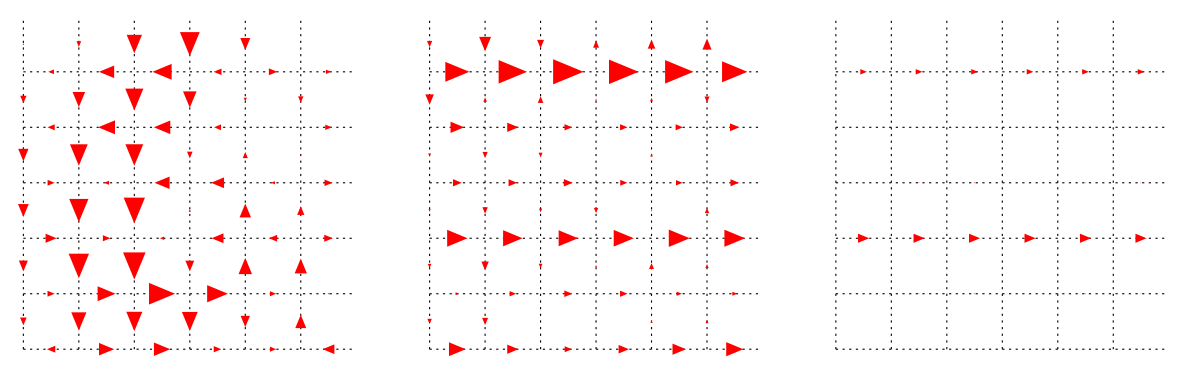

Figure 10. Map of the local persistent currents in a given sample for small (left), intermediate (center) and large (right) values of $r_{s}$.

If one looks at the distribution of the angles $\theta_{i}$ of the local currents, one can see in Fig.11 that the currents are randomly scattered without interaction, and that they become aligned when one goes to the strong coupling limit. The ensemble average value $<|\theta|>$ allows us to quantify the progressive change. If $p(\theta)=1 /(2 \pi),<|\theta|>=\pi / 2$, a value obtained for the low ratios $r_{s}$. At large $r_{s},\left\langle|\theta|>\rightarrow 0\right.$. The ratio $r_{s}$ at which the local currents cease to be oriented at random is consistent with the critical ratio $r_{s}^{F}$ where the $\mathrm{HF}$ approximation breaks down. 

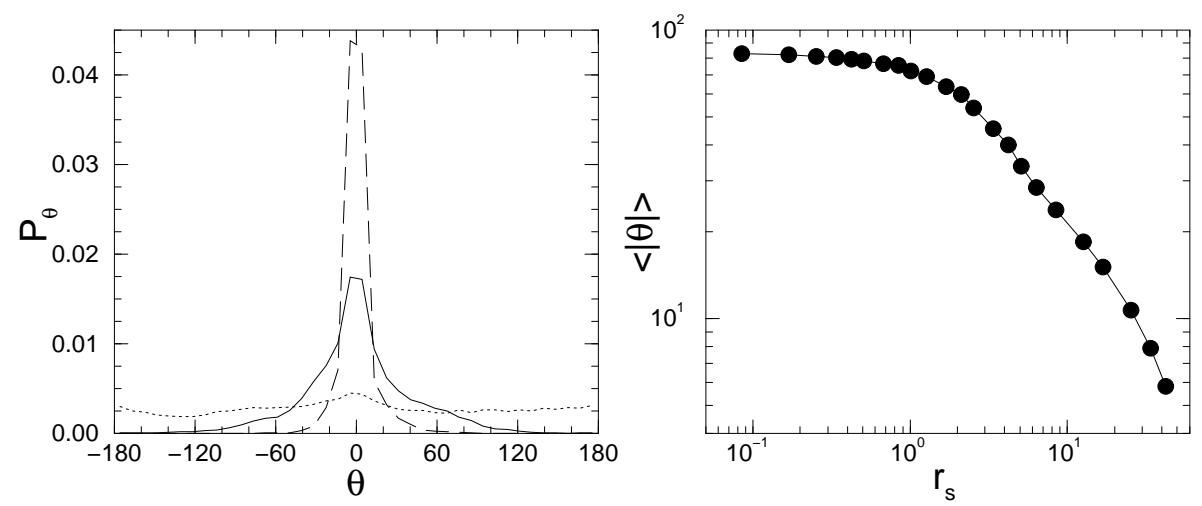

Figure 11. Left: Distribution $P_{\theta}$ of the GS local current angles for $r_{s}=0$ (dotted line), $r_{s}=6.3$ (full line) and $r_{s}=42$ (dashed line). Right: Ensemble average angle $<|\theta|>$ as a function of $r_{s}$.

By studying the average amplitude of the local currents, one can see in Fig. 12 that $\left\langle J_{i}\right\rangle$ is essentially independent of $r_{s}$ up to a second threshold $r_{s}^{W} \approx 10$ which exceeds $r_{s}^{F}$. Moreover, comparing in Fig. 12 the GS average crystallization parameter $\langle\gamma\rangle$ and $\left\langle J_{i}\right\rangle$, on can see that the suppression of the persistent currents coincides with the formation of a solid Wigner molecule inside the disordered clusters.

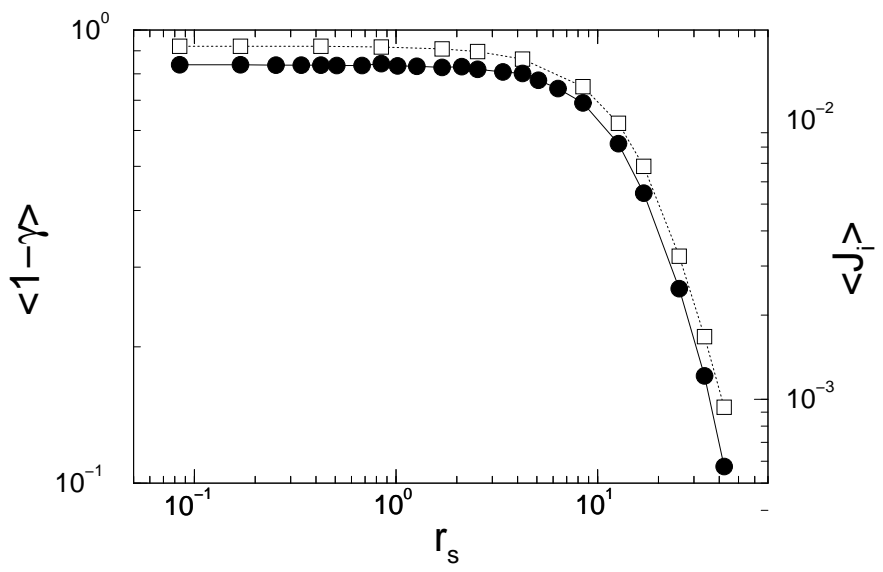

Figure 12. Averages of the GS crystallization parameter $\langle 1-\gamma\rangle$ (left scale, empty symbols) and GS local current amplitude $\left\langle J_{i}\right\rangle$ (right scale, filled symbols) as a function of $r_{s}$. 
The response of the ground state to an enclosed Aharonov-Bohm flux shows us that an intermediate correlated regime takes place between the Fermi limit and the Wigner limit, when typically $5<r_{s}<10$.

\subsection{LOW ENERGY EXCITATIONS}

In Ref. [45], the low energy excitations of the same clusters have been studied, notably their statistics when the microscopic configurations of the random substrate are changed. For intermediate ratios $r_{s}$, the GS and the 8 first following low energy excitations are characterized by oriented non random persistent currents and do not exhibit Wigner-Dyson (W-D) spectral statistics. Above those states, when the excitation energy $\epsilon$ exceeds an energy of the order of the Fermi energy $\epsilon_{F}$, the local currents become randomly oriented and the levels obey W-D statistics. Incidentally, let us note that the metallic behavior observed for intermediate couplings disappears also when the temperature exceeds a temperature of the order of the Fermi temperature.
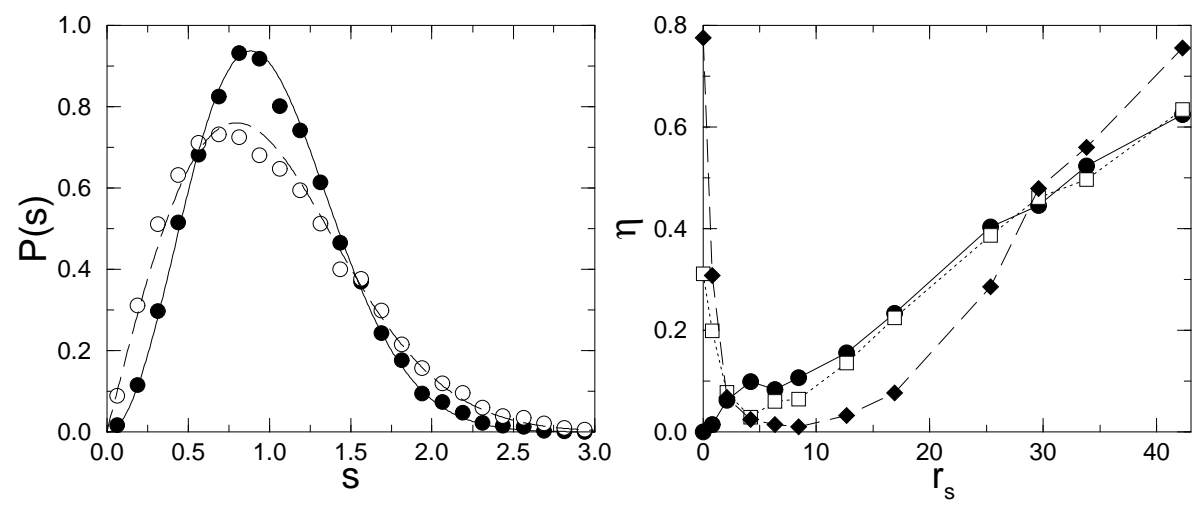

Figure 13. Left: Spacing distribution $P(s)$ for $r_{s}=6.3$ when $\phi=0$ (०) and $\phi=\pi / 2$ (•) for energy levels above the 9 first levels (excitation energies $1.4<\epsilon / \epsilon_{F}<1.9$ ), compared to $P_{O}^{W}(s)$ (dashed line) and $P_{U}^{W}(s)$ (continuous line). Right: Spectral parameter $\eta_{U}$ as a function of $r_{s}$ for the first excitation $s_{0}$ (circles), $s_{2}-s_{4}$ (squares) and $s_{10}-s_{20}$ (diamonds).

In Fig. 13 (left) one can see that the spacing distribution $P(s)$ calculated for $r_{s}=6.3$ using the low energy levels except the 9 first levels $(n=$ $10, \ldots, 20)$, is given by the Wigner surmise, with an orthogonal-unitary crossover when one turns on an Aharonov-Bohm flux $\phi=0 \rightarrow \pi / 2$. This corresponds to excitation energies $1.4<\epsilon / \epsilon_{F}<1.9$. Taking $\phi=\pi / 2$, the variation of the spectral parameter $\eta_{U}$ as a function of $r_{s}$ is given for 
the successive level spacings in Fig. 13. The first excitation is described by the Wigner surmise $\left(\eta_{U}=0\right)$ without interaction but becomes more and more Poissonian when $r_{s}$ increases. The spacings characterizing the levels with $n=10, \ldots, 20$ have an opposite behavior. For $r_{s}=0$, those excitations being the sum of more than one single-electron excitation are essentially uncorrelated, but become correlated for intermediate $r_{s}$ $\left(\eta_{U} \approx 0\right)$ before being again uncorrelated at larger $r_{s}$.

In summary, when one considers the low energy spectral statistics, a complementary signature of an intermediate regime is obtained, given by W-D statistics and randomly oriented local persistent currents outside the 9 first states for which the absence of W-D statistics for intermediate $r_{s}$ is accompanied by a non random orientation of the persistent current angles $\theta$ (see Ref. [45]). This behavior does not appear for weak and strong couplings, where the low energy spectral correlations decrease as the excitation energy increases.

\subsection{INTERMEDIATE LIQUID-SOLID REGIME IN THE CLEAN LIMIT}

The fact that the 9 first states do not display quantum ergodicity for intermediate coupling and weak disorder suggests the existence of 9 low energy collective excitations. A collective motion cannot be due to impurity scattering and should come from the corresponding clean limit. This limit has been investigated in Ref. [46].

When $W=0$, one has a single system which remains invariant under rotation of angle $\pi / 2$ and under translations and reflections along the longitudinal $x$ and transverse $y$ directions. Invariance under translations implies that the momentum $K$ is a good quantum number which remains unchanged when $U$ varies. The symmetries imply that the states are fourfold degenerate if $K \neq 0$ and can be non degenerate if $K=0$.

When $U=0$, the states are $N_{H}$ plane wave Slater determinants (SDs) $d_{k(4)}^{\dagger} d_{k(3)}^{\dagger} d_{k(2)}^{\dagger} d_{k(1)}^{\dagger} \mid 0>$, where $d_{k(p)}^{\dagger}$ creates a particle in a state of momentum $k(p)=2 \pi\left(p_{x}, p_{y}\right) / L\left(p_{x, y}=1, \ldots, L\right)$ and $\mid 0>$ is the vacuum state. For $N=4$ and $L=6, N_{H}=58905$. The low energy eigenstates are given by the following plane wave SDs:

- 4 degenerate ground states (GSs) $\mid K_{0}(\beta)>(\beta=1, \ldots, 4)$ of energy $E_{0}(U=0)=-13 t$ and of momenta $K_{0}=(0, \pm \pi / 3)$ and $( \pm \pi / 3,0)$.

- 25 first excitations of energy $E_{1}(U=0)=-12 t$ out of which 4 plane wave SDs $\mid K_{1}(\beta)>$ will play a particular role for describing the intermediate GS. They correspond to a particle at an energy $-4 t$ with $k(1)=(0,0)$, two particles at an energy $-3 t$ and a fourth 
particle of energy $-2 t$ with momenta such that $\sum_{j=2}^{4} k(j)=0$. One has $k(2)=(0, \pm \pi / 3), k(3)=( \pm \pi / 3,0)$ and $k(4)=(\mp \pi / 3, \mp \pi / 3)$ or $k(2)=(0, \mp \pi / 3), k(3)=( \pm \pi / 3,0)$ and $k(4)=(\mp \pi / 3, \pm \pi / 3)$.

- 64 second excitations $\mid K_{2}(\alpha)>$ of energy $E_{2}(U=0)=-11 t$.

- 180 third excitations $\mid K_{3}(\alpha)>$ of energy $E_{3}(U=0)=-10 t$.

- 384 fourth excitations of energy $E_{4}(U=0)=-9 t$ out of which 16 plane waves SDs $\mid K_{4}(\delta)>$ will play a particular role for describing the intermediate GS. They are given by the condition that the total momentum is zero, which selects 20 SDs out of which 4 where the single particle state of energy $-3 t$ is not occupied do not contribute. The $\mid K_{4}(\delta)>$ are 16 SDs of energy $-9 t$, given by 8 SDs where the particles have energies $-4 t,-3 t,-2 t, 0 t$ respectively and by 8 other SDs where the particles have energies $-3 t,-3 t,-2 t,-t$ respectively.

When $t=0$, the states are $N_{H}$ Slater determinants $c_{i}^{\dagger} c_{j}^{\dagger} c_{k}^{\dagger} c_{l}^{\dagger} \mid 0>$ built out from the site orbitals. The configurations $i j k l$ correspond to the $N_{H}$ different patterns characterizing 4 different sites of the $6 \times 6$ square lattice. The low energy part of the spectrum is made of the following site SDs:

- 9 squares $\mid S_{0}(I)>(I=1, \ldots, 9)$ of side $a=3$ and of energy $E_{0}(t=0) \approx 1.80 U$.

- 36 parallelograms $\mid S_{1}(I)>$ of sides $(3, \sqrt{10})$ and of energy $\approx 1.85 U$.

- 36 other parallelograms $\mid S_{2}(I)>$ of sides $(\sqrt{10}, \sqrt{10})$ and of energy $\approx 1.97 U$.

- 144 deformed squares $\mid S_{3}(I)>$ obtained by moving a single site of a square $\mid S_{I}>$ by one lattice spacing and of energy $\approx 2 U$.

For the first low energy states, the crossover from the $U=0$ eigenbasis towards the $t=0$ eigenbasis is shown in Fig. 14 when one increases the ratio $r_{s}$. If we follow the $4 \mathrm{GSs} E_{0}\left(r_{s}=0\right)\left(K_{0} \neq 0\right)$, one can see a first level crossing at $r_{s}^{F} \approx 9.3$ with a non degenerate state $\left(K_{0}=0\right)$ which becomes the GS above $r_{s}^{F}$, followed by two other crossings with two other sets of 4 states with $K_{I} \neq 0$. When $r_{s}$ is large, 9 states coming from $E_{1}\left(r_{s}=0\right)$ have a smaller energy than the 4 states coming from $E_{0}\left(r_{s}=0\right)$. The degeneracies ordered by increasing energy become $(1,4,4,4, \ldots)$ instead of $(4,25,64, \ldots)$ for $r_{s}=0$. Since the degeneracies are $(9,36,36, \ldots)$ when $t=0$, these 9 states give the 9 square molecules 


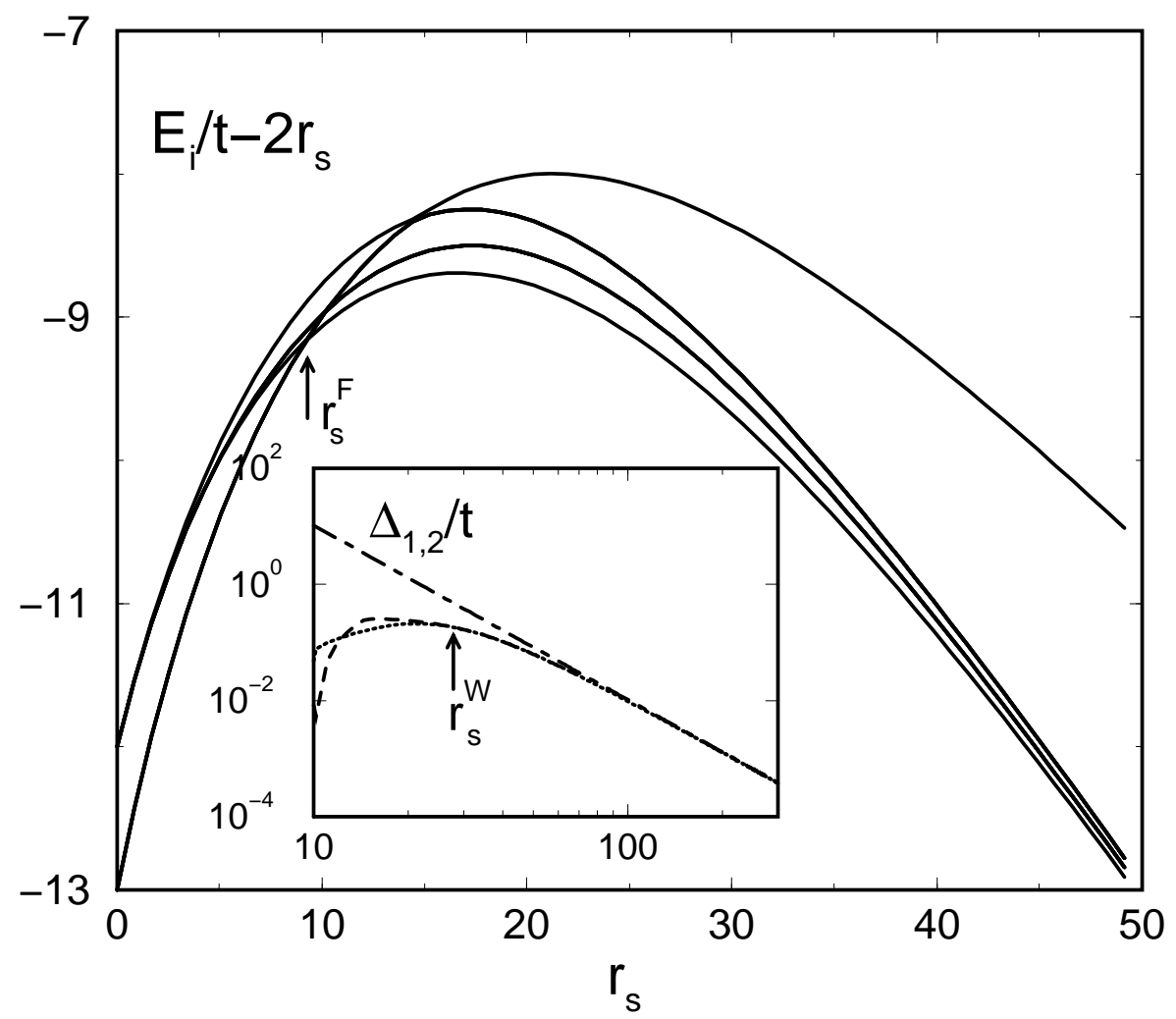

Figure 14. As a function of $r_{s}$, low energy part of the spectrum exhibiting a GS level crossing at $r_{s}^{F}$. Inset: two first level spacings $\Delta_{1} / t$ (dashed) and $\Delta_{2} / t$ (dotted) which become equal at $r_{s}^{W}$ and the perturbative result $\Delta_{1} / t=\Delta_{2} / t \approx 10392 / r_{s}^{3}$ valid when $r_{s} \rightarrow \infty$ (dot-dashed).

$\mid S_{0}(I)>$ when $r_{s} \rightarrow \infty$. When $r_{s}^{-1}$ is very small, the first 9 states correspond to a single massive molecule free to move on a restricted $3 \times 3$ lattice, the single non frozen degree of freedom in this limit being the location $R_{I}$ of the center of mass of the $\mid S_{0}(I)>$. One has an effective hopping term $T \propto t r_{s}^{-3}$ when $N=4$ and the total momentum is quantized $\left(K_{l}(I)=2 \pi p_{l} / 3\right.$ and $K_{t}(I)=2 \pi p_{t} / 3$ being its longitudinal and transverse components respectively with $\left.p_{l, t}=1,2,3\right)$. For a square lattice at a filling factor $1 / 9$, the $R_{I}$ are indeed located on a periodic $3 \times 3$ square lattice. This is an important simplification of our model. This gives 9 states of kinetic energies given by $-2 T\left(\cos K_{l}(I)+\cos K_{t}(I)\right)$. The kinetic part of the low energy spectrum is then $-4 T,-T,+2 T$ with 
degeneracies 1,4,4 respectively. This structure with two equal energy spacings $\Delta_{1}$ and $\Delta_{2}$ appears (inset of Fig. 14) when $r_{s}$ is larger than the crystallization threshold $r_{s}^{W} \approx 28$. Above $r_{s}^{W}$, to create a defect in the rigid molecule costs a high energy available in the $10^{\text {th }}$ excitation only. We have seen in the previous section that the 9 first levels do not obey Wigner-Dyson statistics at intermediate $r_{s}$ when a random potential is added, in contrast to the following levels. The study of the clean limit gives us the explanation. The two characteristic thresholds $r_{s}^{F}$ (level crossing) and $r_{s}^{W}$ (9 first states having the structure of the spectrum of a single massive molecule free to move on a $3 \times 3$ square lattice) can also be detected by other methods given in Ref. [46].

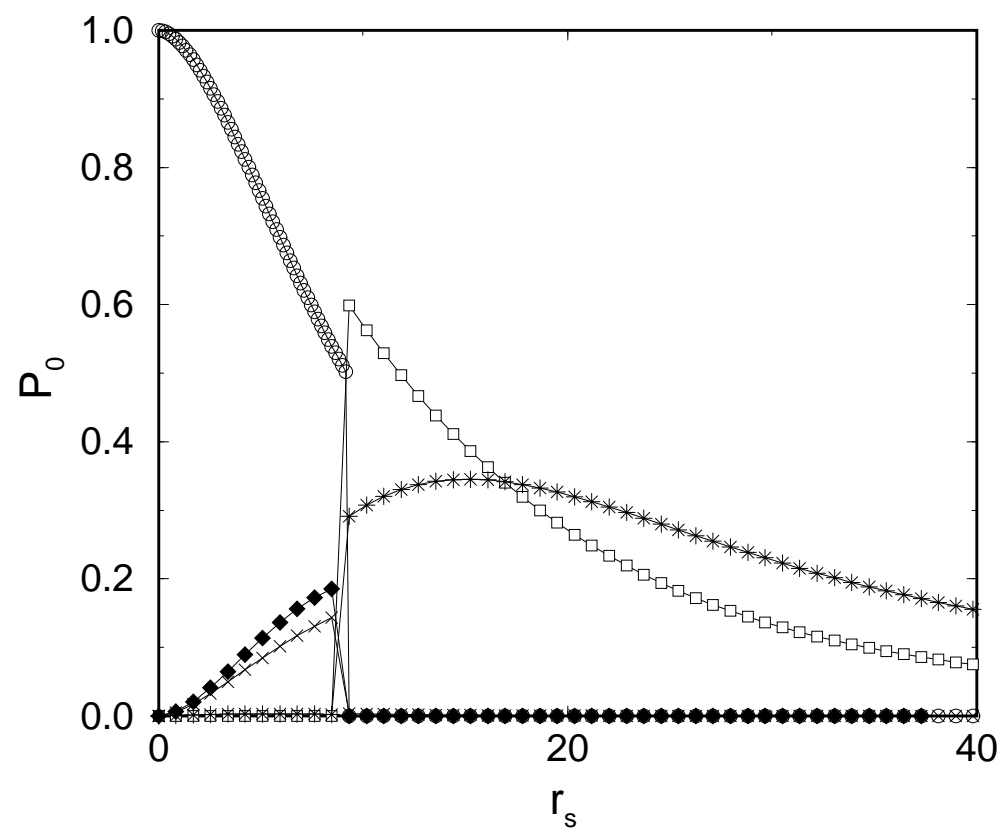

Figure 15. Ground state projections $P_{0}\left(r_{s}\right)$ onto a few plane wave SDs, given by the $4 \mid K_{0}(\beta)>$ (empty circle), the $4 \mid K_{1}(\beta)>$ (empty square), the $64 \mid K_{1}(\alpha)>$ (filled diamond), the $180 \mid K_{2}(\alpha)>(\times)$, the $16 \mid K_{4}(\delta)>$ (asterisk) respectively, as a function of $r_{s}$.

To understand further the nature of the intermediate GS, we have projected the GS wave functions $\mid \Psi_{0}\left(r_{s}\right)>$ over the low energy eigenvectors of the two eigenbases valid for $U / t=0$ (Fig. 15) and for $t / U=0$ (Fig. 17) respectively. 
Let us begin by studying the GS structure in the $U=0$ eigenbasis. Below $r_{s}^{F}$, each of the 4 GSs $\mid \Psi_{0}^{\alpha}\left(r_{s}\right)>$ with $K_{0} \neq 0$ has still a large projection

$$
P_{0}\left(r_{s}, 0\right)=\sum_{\beta=1}^{4}\left|<\Psi_{0}^{\alpha}\left(r_{s}\right)\right| K_{0}(\beta)>\left.\right|^{2}
$$

over the 4 non interacting GSs. There is no projection over the 25 first excitations and smaller projections $P_{0}\left(r_{s}, 2\right)$ and $P_{0}\left(r_{s}, 3\right)$ over the 64 second and 180 third excitations of the non interacting system. Above $r_{s}^{F}$, the non degenerate GS with $K_{0}=0$ has a large projection

$$
P_{0}\left(r_{s}, 1\right)=\sum_{\beta=1}^{4}\left|<\Psi_{0}\left(r_{s}\right)\right| K_{1}(\beta)>\left.\right|^{2}
$$

which is equally distributed over the 4 excitations $\mid K_{1}(\beta)>$ of momentum $K_{1}=0$ and a second significant contribution

$$
P_{0}\left(r_{s}, 4\right)=\sum_{\delta=1}^{16}\left|<\Psi_{0}\left(r_{s}\right)\right| K_{4}(\delta)>\left.\right|^{2}
$$

given by its projection onto the 16 plane wave SDs $\mid K_{4}(\delta)>$ belonging to the fourth excitation of the non interacting system. Above $r_{s}^{F}$, its projections onto the $4\left|K_{0}(\beta)\right\rangle$, the 21 other first excitations and the second and third excitations of the non interacting system are zero or extremely negligible.

The total GS projection

$$
P_{0}^{t}\left(r_{s}\right)=P_{0}\left(r_{s}, 1\right)+P_{0}\left(r_{s}, 4\right)
$$

onto the $4 \mid K_{1}(\beta)>$ and $16 \mid K_{4}(\delta)>$ is given in Fig. 16 when $r_{s}>r_{s}^{F}$. This shows us that a large part of the system remains an excited liquid above $r_{s}^{F}$, given by a special rule of occupation of the one particle plane wave states. The occupation of the one body states is very different from the usual Pauli rule after the level crossing $r_{s}^{F}$. A necessary, but non sufficient condition for a plane wave SD to significantly contribute to the zero momentum GS is of course to have a zero total momentum. Those projections decrease as $r_{s}$ increases and become negligible in the strong coupling limit. A complete GS description in this limit will require more and more plane wave SDs.

We now study the GS projections $P_{\infty}$ onto the $t=0$ eigenbasis. The GS projection

$$
P_{\infty}\left(r_{s}, 0\right)=\sum_{I=1}^{9}\left|<\Psi_{0}^{\alpha}\left(r_{s}\right)\right| S_{0}(I)>\left.\right|^{2}
$$




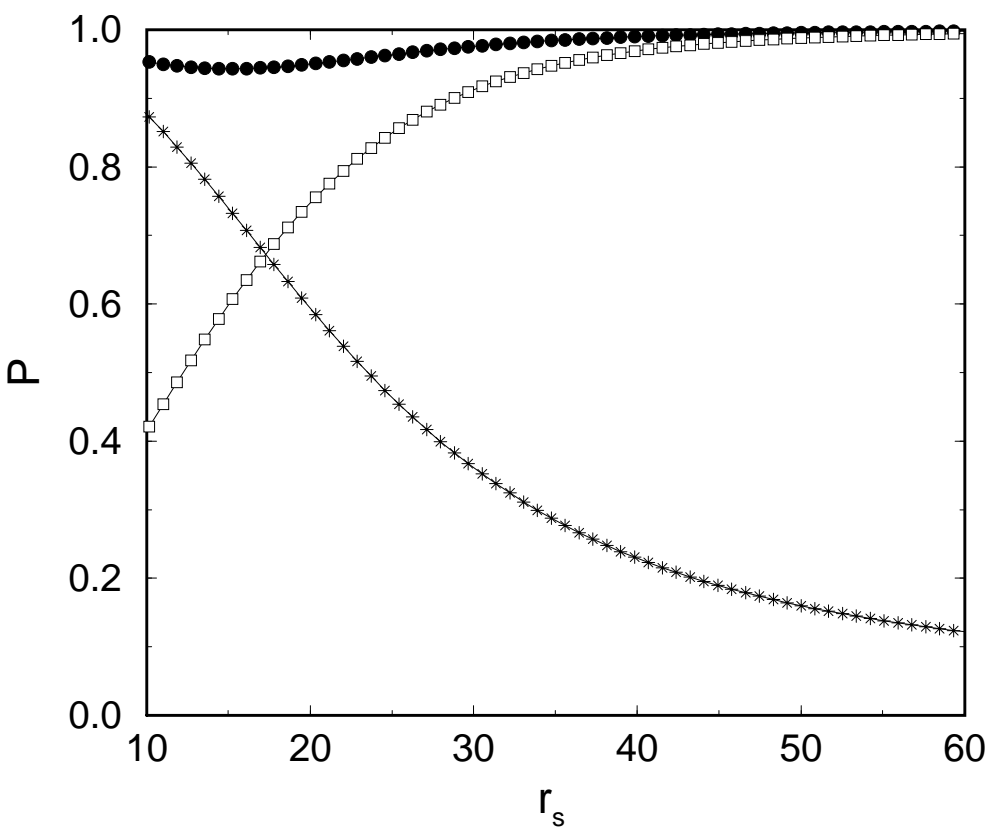

Figure 16. Ground state projection $P_{0}^{t}\left(r_{s}\right)$ (asterisk) and $P_{\infty}^{t}\left(r_{s}\right)$ (empty square) onto the subspace spanned by the low energy plane wave and site SDs respectively, and total GS projection $P$ (filled circle) onto the re-orthonormalized basis using the low energy eigenvectors of the two limiting bases.

onto the 9 square site SDs $\mid S_{0}(I)>$ is given in Fig. 17, together with the GS projection $P_{\infty}\left(r_{s}, J\right)$ onto the site SDs corresponding to the $J^{\text {th }}$ degenerate low energy excitations of the $t=0$ system. The total GS projection

$$
P_{\infty}^{t}\left(r_{s}\right)=\sum_{p=0}^{3} P_{\infty}\left(r_{s}, p\right)
$$

onto the 9 squares $\mid S_{0}(I)>$, the 36 parallelograms $\mid S_{1}(I)>$, the 36 other parallelograms $\mid S_{2}(I)>$ and the 144 deformed squares $\mid S_{3}(I)>$ is given in Fig. 16 when $r_{s}>r_{s}^{F}$. This shows us that the ground state begins to be a floppy solid also above $r_{s}^{F}$. When $r_{s}$ increases, $P_{\infty}\left(r_{s}, 0\right)$ goes to one, and the ground state is a simple rigid square Wigner molecule.

The site SDs and plane wave SDs are not orthonormal. After reorthonormalization, the total projection $P$ of $\mid \Psi_{0}\left(r_{s}\right)>$ over the subspace spanned by the $4 \mid K_{1}(\beta)>$ and $16 \mid K_{4}(\delta)>$ and 225 site SDs of lower electrostatic energies (9 squares, $36+36$ parallelograms, 144 


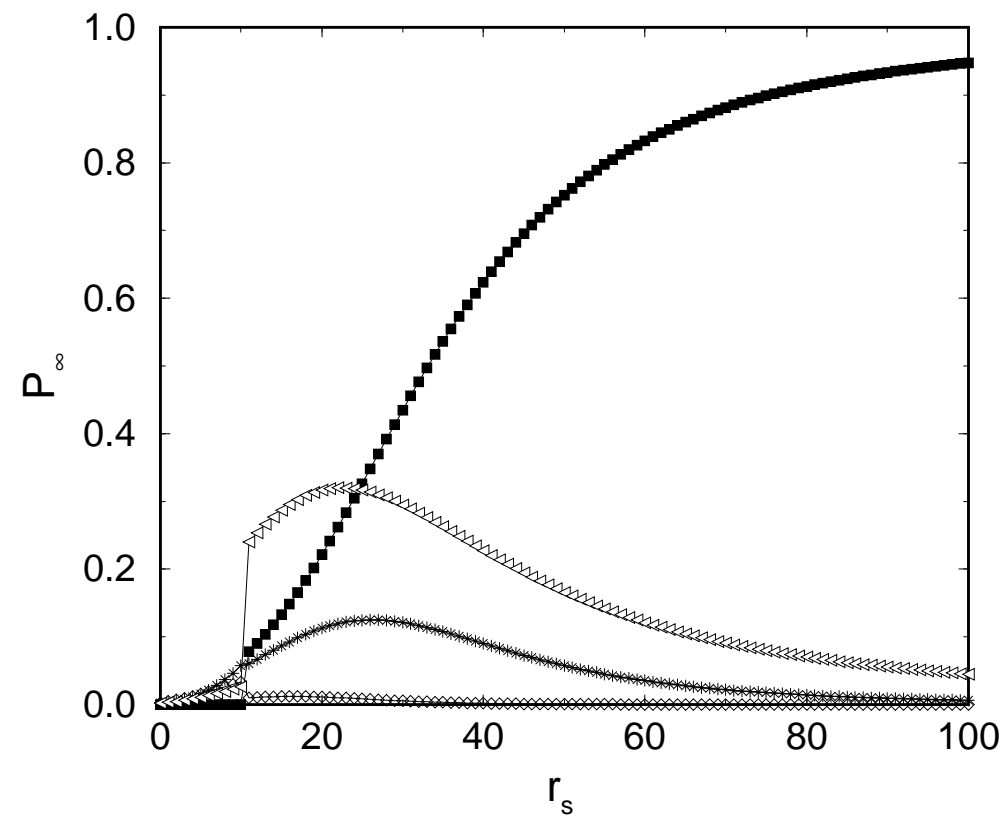

Figure 17. Ground state projection $P_{\infty}\left(r_{s}\right)$ onto a few site SDs, given by the 9 squares $\mid S_{0}(I)>$ (filled square), the 36 parallelograms $\mid S_{1}(I)>$ (asterisk), the 36 parallelograms $\mid S_{2}(I)>$ (diamond), and the 144 deformed squares $\mid S_{3}(I)>$ (left triangle) respectively, as a function of $r_{s}$.

deformed squares) are given in Fig. 16. One can see that $\mid \Psi_{0}\left(r_{s}\right)>$ is almost entirely located inside this very small part of a huge Hilbert space for intermediate $r_{s}$, spanned by no more than 245 low energy SDs of different nature, and adapted to describe a solid entangled with an excited liquid.

From the study of the GS projections emerges the conclusion that a minimal description of the intermediate GS requires to combine the low energy states of the two limiting eigenbases. In this sense, the GS is neither solid, nor liquid, but rather the quantum superposition of those two states of matter. This is strongly reminiscent of the conjecture proposed in Ref. [23] for the quantum melting of solid Helium in three dimensions. It suggests possible improvements of the trial GS to use for intermediate $r_{s}$ in variational or path integral quantum Monte Carlo approaches. Instead of using Jastrow wave functions improving the plane wave SDs for the liquid or the site SDs for the solid, or their nodal surfaces, it will be interesting to study if a combination of the two, with a 
more complex nodal structure, and describing a solid-liquid regime does not lower the GS energy for intermediate $r_{s}$. A positive answer would confirm that an unnoticed intermediate solid-liquid phase does exist in the thermodynamic limit for fermionic systems in two dimensions.

\subsection{MAGNETIC SIGNATURE OF THE INTERMEDIATE REGIME FOR WEAK DISORDER}

We return to the study of weakly disordered samples when the spin degrees of freedom are included. Their role and the consequences of an applied parallel magnetic field which align only the spins without inducing orbital effects, have been the subject of Ref.[47]. A statistical ensemble of matrices having the structure given in Fig. 7 have been studied for $W=5, N=4$ and $L=6$ providing complementary signatures of a particular intermediate behavior.

When $r_{s}=0$, the two one body states of lowest energy are doubly occupied and $S=0(S=1 / 2$ if $N$ is odd). To polarize the $S=0$ ground state to $S=1$ corresponds to the transition of one electron at the Fermi energy and costs an energy equal to the one body level spacing. $p\left(Q_{1}\right)$ is then given by the spacing distribution $p(s)$ between consecutive one body levels, the Wigner surmise $P_{W}^{O}(s)$ in the diffusive regime. When $r_{s}$ is large, the 4 electrons occupy the four sites $c_{j} j=1, \ldots, 4$ of the square configuration $\mid S_{0}(I)>$ of side $a=3$ with the lowest substrate energy $\sum_{j=1}^{4} v_{c_{j}}$. The ground state in this limit becomes $\left|\Psi_{c}>=\prod_{j=1}^{4} c_{c_{j}, \sigma_{j}}^{\dagger}\right| 0>$ with a spin independent energy $E_{c}$. This square can support $2^{N}=16$ spin configurations. We summarize the main results of a perturbative expansion of $E_{c}$ in powers of $t / U$. The spin degeneracy of $E_{c}$ is removed by terms of order $t(t / U)^{2 a-1}$, which is the smallest order where the 16 spin configurations can be coupled via intermediate configurations allowing a double occupancy of the same site. Therefore, $2 a-1$ is the order where the perturbation begins to depend on $S_{z}$ and $Q_{1}$ as $Q_{2} \propto t(t / U)^{2 a-1} \rightarrow 0$ when $t / U \rightarrow 0$ (we have numerically checked this decay when $\left.r_{s}>100\right)$. Moreover, the correction to $E_{c}$ depending on $S_{z}$ and $\propto t(t / U)^{2 a-1}$ is given by an effective antiferromagnetic Heisenberg Hamiltonian. The $S=0$ ground state for large $r_{s}$ correspond to 4 electrons forming an antiferromagnetic square Wigner molecule. However $Q_{1}$ and $Q_{2}$ are very small when $r_{s}$ is large, and the antiferromagnetic behavior can be an artefact due to the square lattice. Without impurities and in a continuous limit, a quasi-classical WKB expansion [21] valid for very large values of $r_{s}$ shows that 3 particle exchanges dominate, leading to ferromagnetism. Recent Monte-Carlo calculations [20] suggest that 


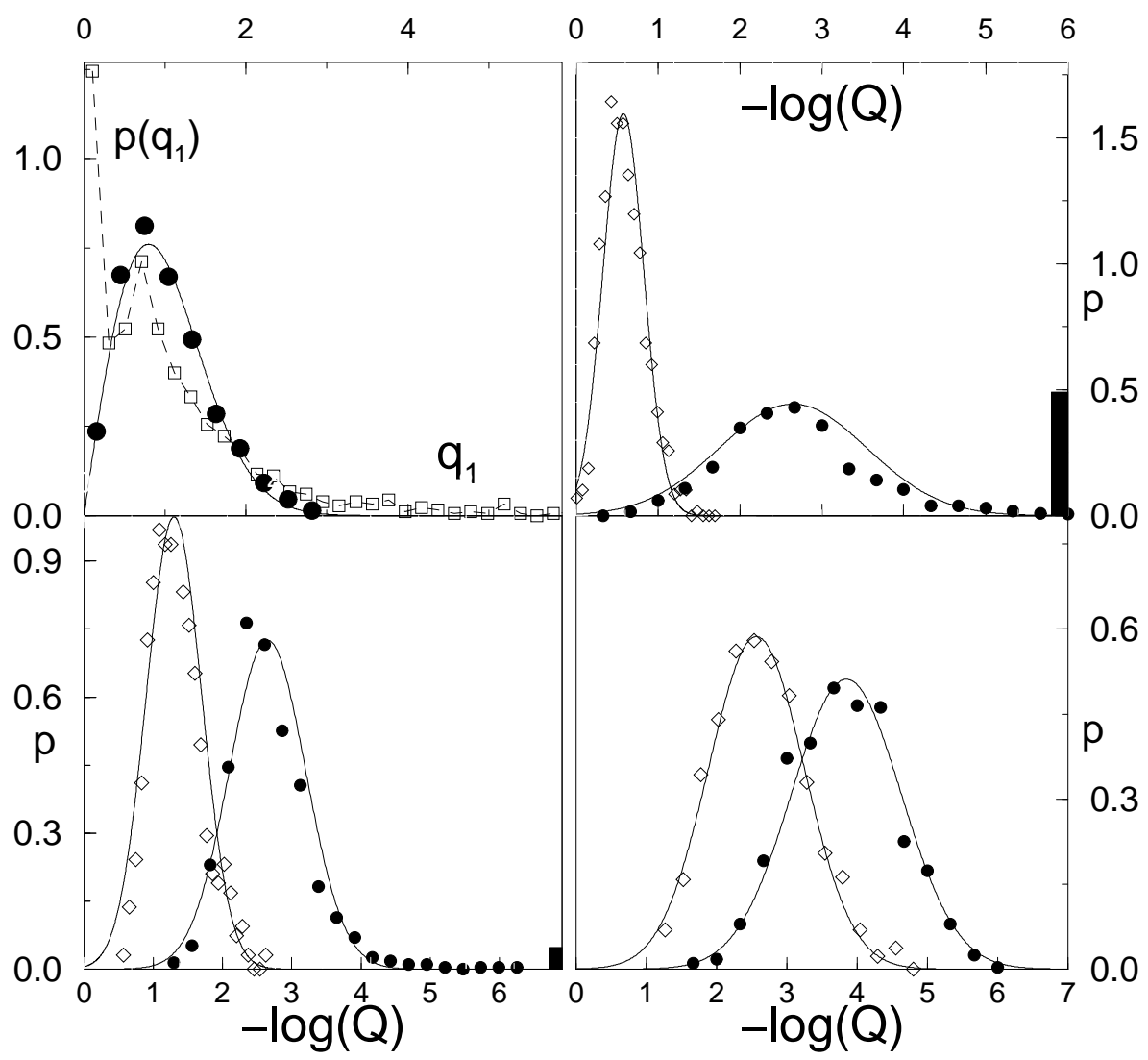

Figure 18. Distributions $p$ of the polarization energies $Q_{1}$ and $Q_{2}$ at different values of $r_{s}$. Upper left: $p\left(q_{1}\right)$ at $r_{s}=0$ (circle) and 2.5 (square) where $q_{1}=Q_{1} /\left\langle Q_{1}\right\rangle$. The continuous line is the Wigner surmise. $p\left(-\log Q_{1}\right)$ (filled circle) and $p\left(-\log Q_{2}\right)$ (empty diamond) at $r_{s}=2.5$ (upper right, right scale) 5.8 (lower left, left scale) and 16.8 (lower right, right scale) respectively. The thick bars (put at right edge of the figures) give the peaks $\delta\left(Q_{1}\right)$ of the bimodal $p\left(Q_{1}\right)$. The continuous lines are normal fits. 
the crystal in the continuous limit becomes a frustrated antiferromagnet closer to the melting point.

The perturbative corrections $\propto t(t / U)^{2 a-1}$ depend on the random variables $v_{i}$ via $\prod_{J=1}^{2 a-1}\left(E_{c}-E_{J}\right)^{-1}$ where the $E_{J}$ are the classical energies of the intermediate configurations. $E_{J}$ is the sum of an electrostatic energy and of a random substrate energy $E_{s}(J)=\sum_{k=1}^{4} v_{J(k)}$. Due to the high order $2 a-1$ of the correction, a normal distribution for $E_{J}$ leads to a log-normal distribution for $\prod_{J=1}^{2 a-1}\left(E_{c}-E_{J}\right)^{-1}$. Therefore $p\left(\Delta_{1}\right)$ and $p\left(\Delta_{2}\right)$ should be log-normal when $r_{s}$ is large.

$p\left(Q_{1}\right)$ is given in Fig. 18 for different $r_{s}$. The expected Wigner surmise takes place for $r_{s}=0$. A small interaction quickly drives $p\left(Q_{1}\right)$ towards a bimodal distribution, with a delta peak at $Q_{1}=0$ and a main peak centered around a non zero value of $Q_{1}$. The delta peak gives the probability to have spontaneously magnetized clusters with $S=1$. The main peak gives the field $B$ necessary to create $S=1$ in a cluster with $S=0$. The logarithmic scale used in Fig. 18 (upper right) underlines the bimodal character of the distribution and confirms that the main peak becomes log-normal when $r_{s}$ is large. The distribution of $Q_{2}$ is not bimodal: a fully polarized cluster has never been seen when $B=0 . Q_{2}$ becomes also log-normally distributed when $r_{s}$ is large.

In Fig. 19, the fraction $M$ of clusters with $S=1$ at $B=0$ is given as a function of $r_{s}$. One can see the mesoscopic Stoner instability (see appendix) taking place at $r_{s} \approx 0.35$. The Stoner mechanism should eventually give fully polarized electrons. This is not the case, the increase of $M$ breaks down when $r_{s}=r_{s}^{F S} \approx 2.2$, a value where the Stoner mechanism and hence the HF approximation break down. In the same clusters, we have seen that the HF approximation fails to describe the persistent currents of the fully polarized sub-block of $\mathcal{H}$ (spinless fermions) when $r_{s}>r_{s}^{F P} \approx 5 . r_{s}^{F P}$ takes a smaller value $r_{s}^{F S}$ when the spin degrees of freedom are included. Above $r_{s}^{F S}, M$ regularly decreases to reach a zero value for $r_{s}^{W S} \approx 9$ where an antiferromagnetic square molecule is formed. In the intermediate regime, there is a competition between the Stoner ferromagnetism and the Wigner antiferromagnetism. Since the $S=0$ clusters are characterized by log-normal distributions, the ensemble averages $<\log Q_{1}>$ and $<\log Q_{2}>$ (without taking into account the $S=1$ spontaneously magnetized clusters) define the typical fields $B$ necessary to yield $S=1$ or $S=2$ in a $S=0$ cluster. Fig. 19 provides two magnetic signatures confirming the existence of a novel intermediate regime between the Fermi glass $\left(r_{s}<r_{s}^{F S}\right)$ and the Wigner glass $\left(r_{s}>r_{s}^{W S}\right):<\log Q_{1}>$ becomes roughly independent of $r_{s}$, while $Q_{2} \propto r_{s}^{-2}$. 


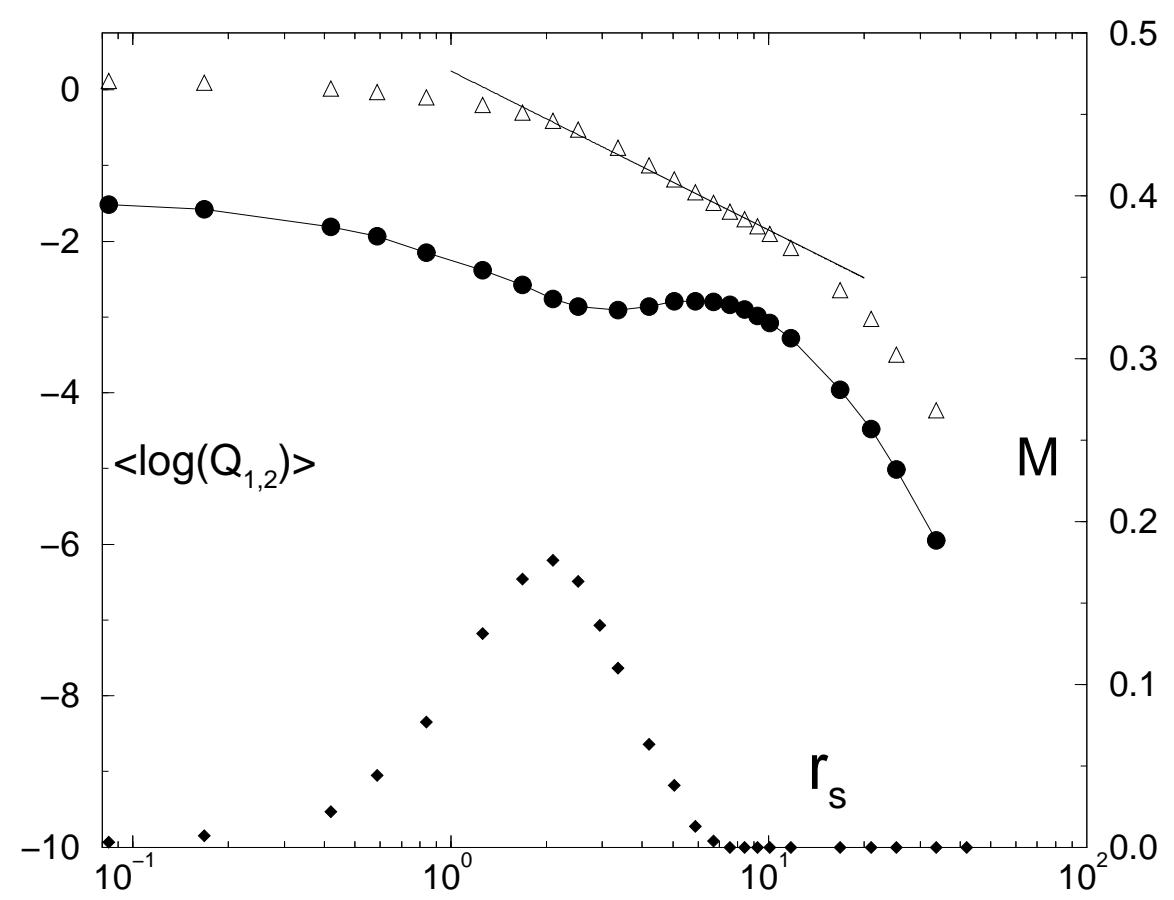

Figure 19. As a function of $r_{s}$, fraction $M$ of clusters with $S=1$ at $B=0$ (filled diamond, right scale), partial $<\log Q_{1}>$ (filled circle, left scale) and total $<\log Q_{2}>$ (empty triangle, left scale) energies required to polarize $S=0$ clusters to $S=1$ and $S=2$ respectively. The straight line corresponds to $0.25-2 \log r_{s}$. 


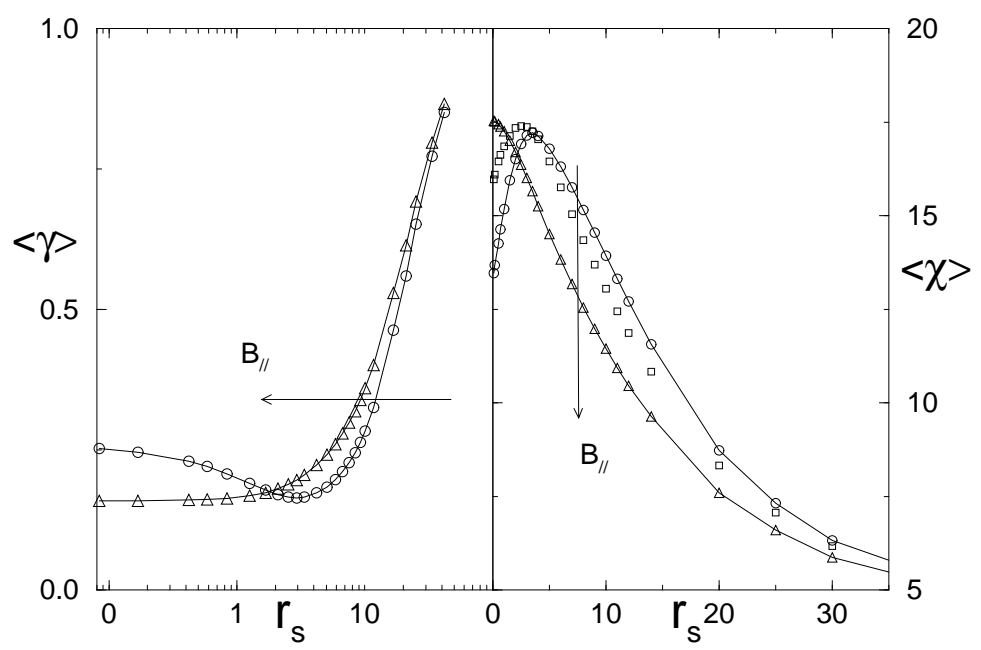

Figure 20. As a function of $r_{s}$, ensemble averages of the crystallization parameter $<\gamma\left(S_{z}\right)>$ (right) and of the numbers of occupied sites $\left\langle\chi\left(S_{z}\right)\right\rangle$ (left). $S_{z}=0$ (circle), $S_{z}=1$ (square) and $S_{z}=2$ (triangle). The arrows indicate the effect of a parallel magnetic field.

The number of occupied sites $\chi\left(S_{z}\right)$ depends on $S_{z}$ for small $r_{s}$ and becomes independent of $S_{z}$ for large $r_{s}$. At $r_{s}=0, \Psi_{0}\left(S_{z}=2,1,0\right)$ occupy respectively $4,3,2$ one body states while the Wigner molecule occupies 4 sites only at large $r_{s}$. The ensemble average $<\chi\left(S_{z}=2\right)>$ shown in Fig. 20 is maximum when $r_{s}=0$ and decays as $r_{s}$ increases, suggesting the absence of delocalization for the polarized system. The non polarized system behaves differently, since $<\chi\left(S_{z}=1,0\right)>$ first increase to reach a maximum $\approx<\chi\left(S_{z}=2, r_{s}=0\right)>$ before decreasing. In Fig.20 one can see also from the curves $\gamma\left(r_{s}\right)$ that charge crystallization is easier when the clusters are polarized than otherwise. The arrows indicated in Fig. 20 underline two consequences of a parallel field $B$ : smaller number of occupied sites and smaller crystallization threshold.

\subsection{NUMERICS VERSUS EXPERIMENTS}

One can question whether the comparison between numerical simulations based on a small lattice model with a few particles and measures using $10^{11}$ electrons per $\mathrm{cm}^{2}$ makes sense. It is nevertheless interesting to point out certains analogies in the obtained thresholds and behaviors.

- The ratios $r_{s}^{W}$ where the rigid Wigner molecule is formed in small clusters are of the order of the ratios $r_{s}^{c}$ where one observes the 
MIT. One finds a value $\approx 10$ for a weakly disordered cluster which increases to larger values when one goes to the clean limit $\left(r_{s}^{W} \approx\right.$ $30-40)$. The $r_{s}^{W}$ numerically obtained for larger disorder $(W=$ $5,10,15)$ in Ref.[42] are roughly independent of $W$ when $W>5$ and reproduce the flat part of the experimental curve sketched in Fig. 5.

- The large magnetoresistance sketched in Fig. 3 is consistent with the reduction of $\chi$ yielded by a parallel magnetic field as indicated in Fig. 20 (right).

- The shift of the critical threshold $r_{s}^{W}$ when one polarizes the electrons with a parallel magnetic field (Fig. 20 - left) is consistent with the shift of the critical densities sketched in Fig. 4.

- A polarization energy $Q_{2} \propto r_{s}^{-2}$ is consistent with the $n_{s}$ dependence of $B_{\text {sat }}$ given in Fig. 3 for similar values $3<r_{s}<9$ [29].

- A threshold at $r_{s}^{F} \approx 3-5$ given by the study of the persistent currents and of the magnetization corresponds to the density at which the metallic behavior ceases to occur, according to Ref. [35].

- It can be argued that the usual small negative magnetoresistance yielded by a perpendicular magnetic field for intermediate $r_{s}$ (Fig. 6 ) would mean that transport is due to the gapless excitations of the intermediate floppy quantum solid. The propagation of such excitations could be reduced by the usual weak localization corrections when one has elastic scattering by impurities.

\subsection{MESOSCOPIC 2D ELECTRON SYSTEMS CONFINED IN HARMONIC TRAPS}

For the important issue of the melting of a macroscopic 2D Wigner glass (crystal without disorder) and a possibly associated 2D-MIT, the study of finite size systems can give hints, but requires to be complemented by a systematic study of the scale dependence of the finite size effects at a given electron density. Before reviewing some results where such a finite size scaling analysis has been done, let us underline that the formation of the mesoscopic Wigner molecule in a few electron system is by itself an important issue. One can use a few electrons [48] confined in a quantum dot or a few ions [49] trapped by electric and magnetic fields. Increasing the size of the trap, a crossover from independent-particle towards collective motion can be observed. Moreover, a localizationdelocalization transition has been observed [50] in a quantum dot using single-electron capacitance spectroscopy and increasing the number of 
trapped electrons. Considering electrons confined in a harmonic trap, and not on a 2D torus, other numerical studies have reached the same crucial conclusion, namely that between the Fermi system and the "classical" rigid molecule, there is an intermediate regime of a floppy weakly formed Wigner molecule. This was shown for instance in an exact study of a two-electron artificial atom [51] and in a Monte Carlo study [52] of a few electron system. In Ref. [52], this new regime corresponds to particles having a radial ordering on shells without correlated intershell rotation. This was attributed to the special symmetry of the harmonic trap and to the imposed density gradient. Our studies show that mesoscopic Wigner crystallization takes also place in two stages when the particles are confined on a $2 \mathrm{D}$ torus, with a uniform electron density.

\section{QUANTUM PHASE TRANSITION FOR WEAK COUPLING AND STRONG DISORDER}

In this Section we consider the case of spinless fermions for strong disorder and low filling factors $N / L^{2}$. In contrast to the previously studied systems, the relevant length scale without interaction, the one-body localization length $L_{1}$, is not only smaller than the system size, but also smaller than the average distance between particles, $d \propto n_{s}^{-1 / 2}$. Truncated bases built out from single-particle wavefunctions or Hartree-Fock orbitals will be used to study larger systems. These approximations remain reliable when the interaction strenght $U$ does not exceed the hopping term $t$, but become uncontrolled in the strongly correlated regime $U \gg t$. They allow us to study the finite size effects in the limit of relatively weak coupling. They show the existence of a second order phase transition between the weak coupling Fermi limit and a new phase appearing above $U_{c} \approx t$ for $W=10-15$. Two numerical evidences of a transition are presented. The first is given by the divergence of a characteristic length of the two dimensional system which allows us to map the finite size data onto a single scaling curve. The second is given by the existence of a size independent distribution of the first excitation at $U_{c}$.

In this limit, the use of the parameter $r_{s}$ becomes questionable, since it is no longer an appropriate measure of the Coulomb to Fermi energy ratio, the one body part of the Hamiltonian being deeply changed by the random substrate. The values $U_{c}$ obtained when $W$ is large give for the considered filling factors critical values $r_{s}^{c}=U_{c} /\left(2 t \sqrt{\pi\left(N / L^{2}\right)}\right.$ of the order of the previously obtained ratios $r_{s}^{F}$ for a weaker disorder. 
However, the use of a dimensionless ratio ignoring $W$ when $W$ is large can be misleading.

\subsection{DIVERGENCE OF A CHARACTERISTIC LENGTH IN TWO DIMENSIONS}
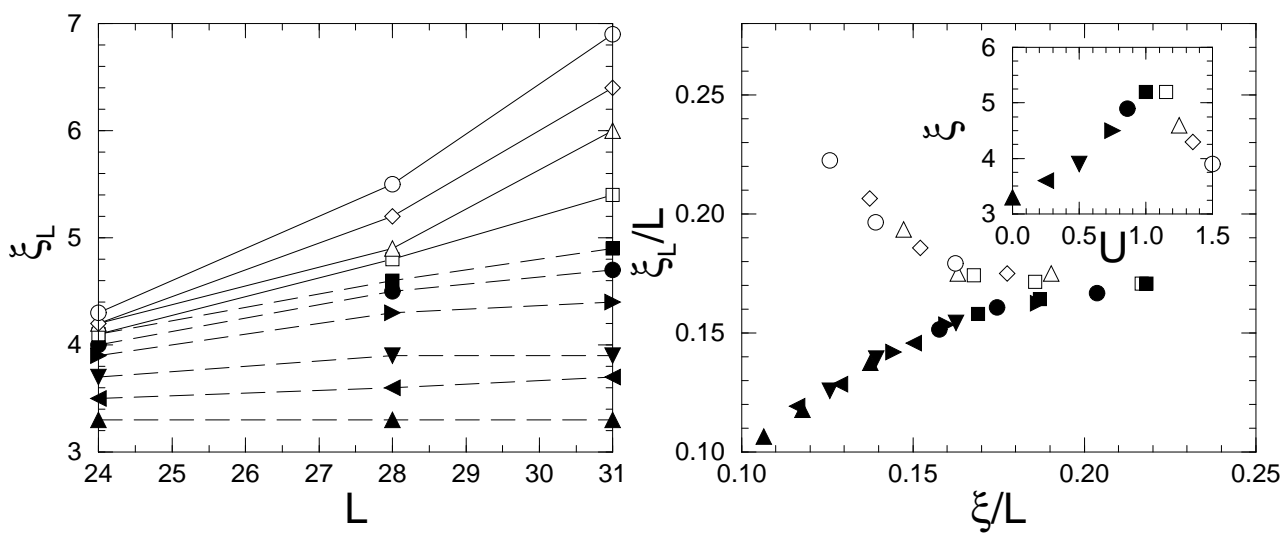

Figure 21. Right: Characteristic length $\xi_{L}$ as a function of $L$ for $U \leq 1$ (filled symbols): 0 (triangles up), 0.25 (triangles left), 0.5 (triangles down), 0.75 (triangles right), 0.85 (circles) and 1 (squares) and $U>1$ (empty symbols): 1.15 (squares), 1.25 (triangles), 1.35 (diamonds) and 1.5 (circles). Left: Ratios $\xi_{L} / L$ mapped onto the scaling curve $f$ as a function of $\xi / L$. The two dimensional scaling length $\xi$ is given in the inset.

From a finite size study [53], one can obtain scaling laws consistent with the divergence of a characteristic length of the two dimensional system at a first lower threshold $U_{c} \approx t$, as in a second order phase transition. For defining a characteristic length of the $N$-body ground state, one considers the change $\delta \rho_{j}$ of the charge density induced by a small change $\delta v_{i}$ of the random potential $v_{i}$ located at a distance $r=$ $|i-j|$. To improve the statistical convergence, one computes the change $\delta \rho(r)=\sum_{j_{y}} \delta \rho_{r, j_{y}}$ of the charge density on the $L$ sites of coordinate $j_{x}=$ $r$ yielded by the change $v_{0, i_{y}} \rightarrow 1.01 v_{0, i_{y}}$ for the $L$ random potentials of coordinate $i_{x}=0$. For a Slater determinant made with $\mathrm{N}$ occupied single particle eigenfunctions $\left(\psi_{\alpha}\right)$, first order perturbation theory gives:

$$
\delta \rho_{j}=2 \delta v_{i} \sum_{\alpha=1}^{N} \sum_{\beta \neq \alpha} \frac{\psi_{\alpha}(i) \psi_{\beta}(i) \psi_{\alpha}(j) \psi_{\beta}(j)}{E_{\beta}-E_{\alpha}} \propto \exp -\frac{2 r}{L_{1}}
$$


the index $\beta$ varying over the one-body spectrum. Therefore, in the absence of interaction, the change $\delta \rho$ remains localized on a scale given by the one-body localization length $\left(\xi_{L} \approx L_{1} / 2\right)$.

Let us study the dependence of $\xi_{L}$ on the interaction $U$ for an ensemble of $5 \times 10^{3}$ clusters, with $N=3,4,5$ particles in square lattices of size $L=24,28,31$ respectively, corresponding to a very low filling factor $n_{e} \approx 5 \times 10^{-3}$. To have Anderson localization inside these sizes we considered a large disorder to hopping ratio $W / t=10$. Therefore the low energy tail of the one body spectrum is made of impurity states trapped at some site $i$ of exceptionally low $v_{i}$. As we are interested in studying the effect of Coulomb repulsion on genuine Anderson localized states we get rid of the band tail. Typically we ignore the $L^{2} / 2$ first one-body levels (but results do not change, provided that the Fermi level is out of the band tail, $\epsilon_{F}>-4 t$ ). From this restricted subset of one-body states we built a basis for the $N$-body problem, truncated to the $N_{H}=10^{3}$ Slater determinants of lowest energy (convergency tests are discussed in Ref. [53]). We note that, for $W / t=10$, the one-body localization length $L_{1} \approx 4$ is smaller than the distance between particles $d \approx 15$.

We checked that $|\delta \rho(r)|$ is reasonably fitted by a log-normal distribution. Therefore it makes sense to characterize the typical strength of the fluctuations by

$$
\delta \rho_{\text {typ }}(r)=\exp <\ln |\delta \rho(r)|>
$$

and extract the length $\xi_{L}$ over which the perturbation is effective from the exponential decay

$$
\delta \rho_{\text {typ }}(r) \propto \exp \left(-r / \xi_{L}\right) .
$$

The size dependence of $\xi_{L}$ is presented in Fig. 21 left, for increasing Coulomb repulsions. One finds the behavior typical of a phase transition: $\xi_{L}$ converges towards a finite value when $U<U_{c}$ (localized phase), diverges linearly as a function of $L$ at $U=U_{c} \approx 1$ (critical point) and diverges faster than linearly when $U>U_{c}$ (delocalized phase). This is exactly the behavior $[54,55]$ which characterizes the one-body Anderson transition in three dimensions.

In Fig. 21 right we verify a usual scaling ansatz [54] inspired by the theory of second-order phase transitions:

$$
\frac{\xi_{L}}{L}=f\left(\frac{L}{\xi}\right)
$$

where we assume that it is possible to map the characteristic length $\xi_{L}$ at the system size $L$ onto a scaling curve $f(L / \xi)$, where $\xi$ is the scaling length characteristic of the infinite two dimensional system. 
All the data of Fig. 21 left can be mapped onto the universal curve $f$ shown in Fig. 21 right, assuming the scaling length $\xi$ given in the inset. When $U<U_{c}$, this length characterizes the localization of the effect of a local perturbation of the substrate in the two dimensional thermodynamic limit. Our data are consistent with a divergence of $\xi$ at a threshold $U_{c} \approx t$.

Very often, additional corrections $\propto L^{-\alpha}$ to the scaling ansatz occurs for small sizes. We point out that our results can be fitted by a simple linear law $\xi_{L}=0.17 L$ for $U=U_{c}$. This is a further indication that the simple ansatz (22) describes scaling for $L \geq 24$, without noticeable additional $L^{-\alpha}$ corrections.

This tells us that the polarized electron system in a highly disordered $2 d$ substrate becomes correlated when $U>U_{c}$ and that the effect of a local perturbation becomes delocalized.

\subsection{SIZE INDEPENDENT DISTRIBUTION OF THE FIRST EXCITATION}

In Ref. [56] we used the configuration interaction method, discussed in appendix, to study the statistics of the first many-body energy spacing. Questions related to the convergence of the method when the HartreeFock basis is truncated are discussed in Ref. [56].

Let us consider the first $N$-body energy levels $E_{i},(i=0,1,2, \ldots)$ for different sizes $L$, with a large disorder to hopping ratio $W / t=15$ imposed to have Anderson localization and Poissonian spectral statistics for the one particle levels at $U=0$ when $L \geq 8$. This corresponds to a strongly disordered limit where the one particle localization length $L_{1}$ is not only smaller than $L$ and the distance $d$ between the particles (as in the previous subsection), but becomes also of the order of the lattice spacing. We considered $N=4,9$, and 16 particles inside clusters of size $L=8,12$, and 16 respectively. This corresponds to a constant low filling factor $n_{e}=1 / 16$. We studied an ensemble of $10^{4}$ disorder configurations.

The first average spacing $\left\langle\Delta E_{0}\right\rangle$ is given in Fig. 22. It exhibits a power law decay as $L$ increases, with an exponent $\beta$ given in the inset. One finds for the first spacing that $\beta$ linearly decreases from $d=2$ to 1 when $U$ increases from 0 to 2.5. The next mean spacings $<\Delta E_{i}>=<E_{i+1}-E_{i}>$ depend more weakly on $U$, as shown in Fig. 22 .

For $U=0$, the distribution of the first spacing $s=\Delta E_{0} /<\Delta E_{0}>$ becomes closer and closer to the Poisson distribution $P_{P}(s)$ when $L$ increases, as it should be for an Anderson insulator. For a larger $U$, the distribution seems to become close to the Wigner surmise $P_{W}(s)$ 


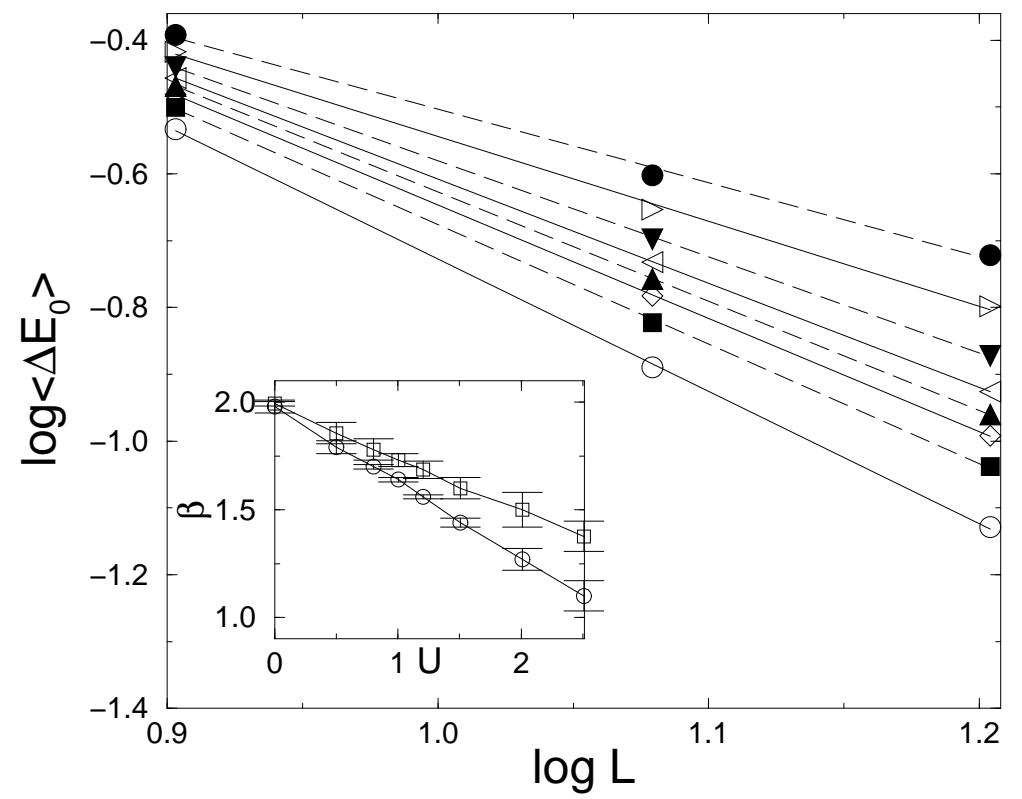

Figure 22. Size dependence of the average gap (first spacing $<\Delta E_{0}>\propto L^{-\beta(U)}$ ), for $W=15$, filling factor $n_{e}=1 / 16$. From bottom to top: $U=0,0.5,0.8,1,1.2,1.5,2,2.5$. Inset: $\beta(U)$ (circles, characterizing $\left\langle\Delta E_{0}\right\rangle$ and squares, characterizing $\left\langle\Delta E_{i}\right\rangle$, with an average over $i=1-3)$.

characteristic of level repulsion in Random Matrix Theory, as shown in Fig. 23 left, for $U=2.5$ and $L=16$. To study how this $P(s)$ goes from Poisson to a Wigner-like distribution when $U$ increases, we use the spectral parameter $\eta_{O}$ which decreases from 1 to 0 when $P(s)$ goes from Poisson to Wigner. In Fig. 23 right, one can see that the three curves $\eta_{O}(U)$ characterizing the first spacing for $L=8,12,16$ intersect at a critical value $U_{c} / t \approx 1.1$. Our data suggest that for $U<U_{c}$ the distribution tends to Poisson in the thermodynamic limit, while for $U>U_{c}$ it tends to a Wigner-like behavior. At the threshold $U_{c}$, there is a size-independent intermediate distribution shown in the inset of Fig. 23 left, exhibiting level repulsion at small $s$ followed by a $\exp (-a s)$ decay at large $s$, with $a \approx 1.52$. Such a size independent distribution is known for characterizing a mobility edge in an one body spectrum. This PoissonWigner transition characterizes only the first spacing, the distributions of the next spacings being quite different. The inset of Fig. 23 right does not show any intersection for the parameter $\eta$ calculated for the second spacing. 

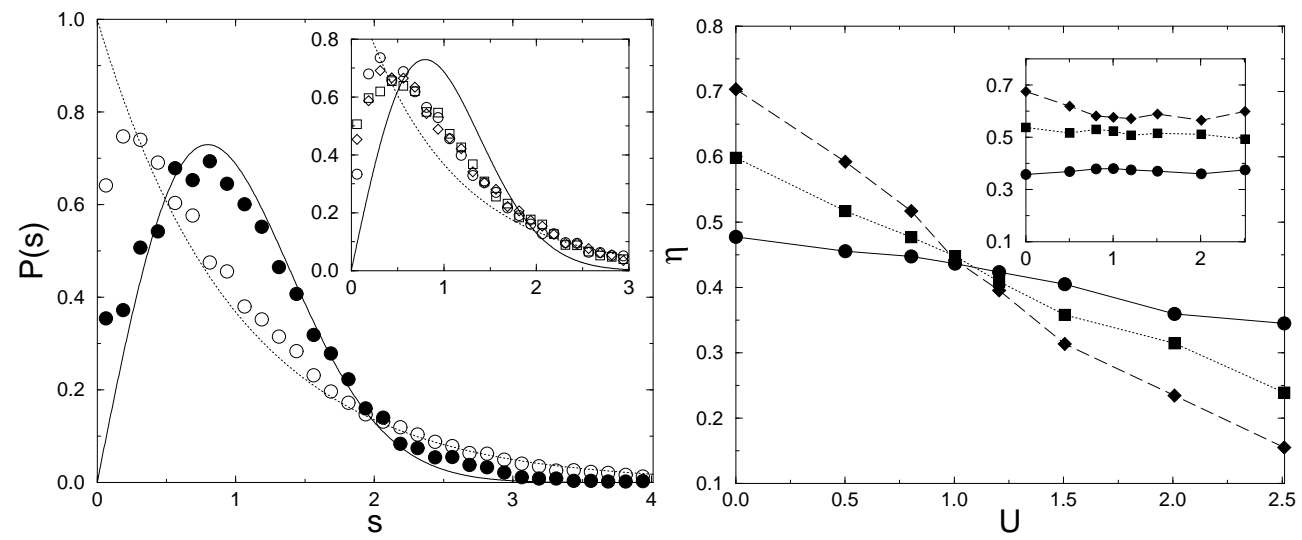

Figure 23. Left: gap distribution $P(s)$ for $U=0$ (empty circles) and $U=2.5$ (filled circles) when $N=16, L=16, W=15$, compared to $P_{P}(s)$ and $P_{W}(s)$. Inset: size invariant $P(s)$ at $U_{c} \approx 1.1 ; L=8$ (circles), 12 (squares), and 16 (diamonds). Right: parameter $\eta_{O}(U)$ corresponding to the first spacing $\Delta E_{0}$ at $L=8$ (circles), 12 (squares), and 16 (diamonds). Inset: $\eta_{O}(U)$ for the second spacing $\Delta E_{1}$.

The observed transition, and the difference between the first spacing and the following ones is mainly an effect of the H-F mean field. For the first spacings, the curves $\eta_{O}$ calculated with the HF data are qualitatively similar. At the mean field level the low energy excitations are particlehole excitations starting from the ground state. The energy spacing between the first and the second excited states is given by the difference of two particle-hole excitations and a Poisson distribution follows if the low energy particle-hole excitations are uncorrelated.

We note that the energy of an electron-hole pair is given by $\epsilon_{j}-\epsilon_{i}-$ $U / r_{i j}$ and the classical argument for the existence of a gap in the single particle density of states does not apply for the many-body spectrum[57]. Therefore the observed opening of a gap for the first energy excitation is a remarkable phenomenon beyond the predictions of the classical Coulomb gap model.

\subsection{CHANGE OF THE INVERSE COMPRESSIBILITY}

In Ref. [56] we studied also the inverse compressibility

$$
\Delta_{2}(N)=E_{0}(N+1)-2 E_{0}(N)+E_{0}(N-1),
$$

i.e. the discretized second derivative of the ground state energy $E_{0}$ with respect to the number $N$ of particles. 
We consider $N=4,9,16$ particles on square lattices of size $L=6,9,12$ respectively, corresponding to a constant filling factor $n_{e}=1 / 9$. Here we focus on the strongly localized regime with disorder strength $W=15$.

The following inverse compressibility data are obtained with the configuration interaction method. We have checked that the residual interaction does not change qualitatively Hartree-Fock results. Indeed HF approximation gives in this weak coupling regime a good estimate of the ground state energy (see Ref. [56]) and the inverse compressibility is a physical observable which only depends on the ground state energies at different number of particles. Neither a precise knowledge of the ground state wavefuction (as in the calculation of persistent currents) nor excited states energies (as in studies of spectral statistics) are required.
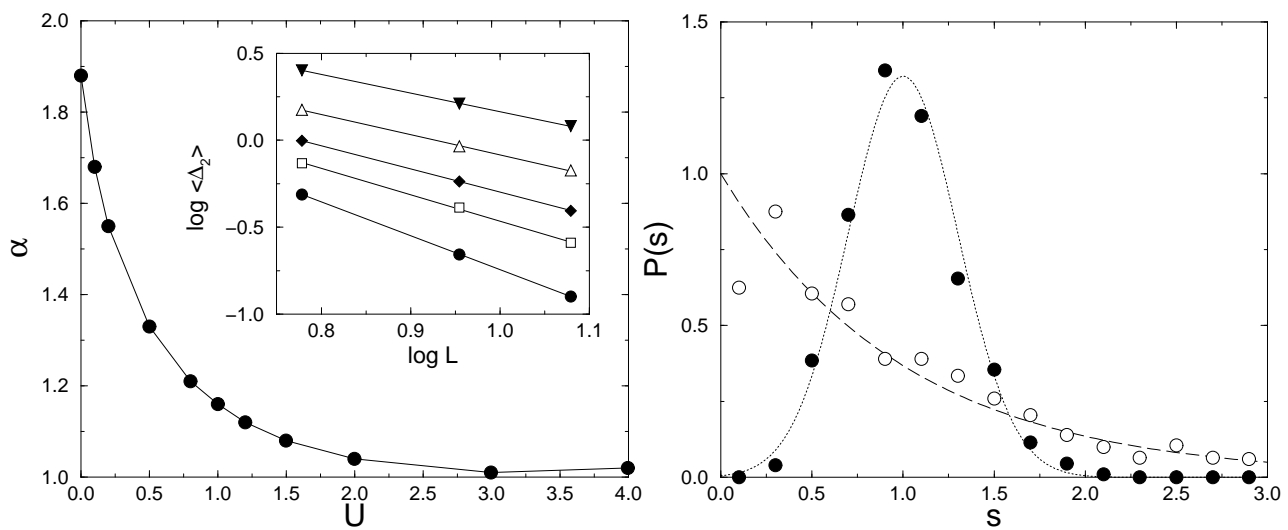

Figure 24. Left: Inset: size dependence of the average inverse compressibility < $\Delta_{2}>$, for $W=15$, filling factor $n_{e}=1 / 9, U=0$ (circles), 0.5 (squares), 1 (diamonds), 2 (triangles up), and 4 (triangles down). Straight lines are power law fits $\left\langle\Delta_{2}(U)>\propto\right.$ $L^{-\alpha(U)}$. Main figure: exponent $\alpha(U)$. Right: distribution of the normalized inverse compressibilities for $N=16, L=12, W=15, U=0$ (empty circles) and $U=3$ (filled circles), fitted by a Gaussian of standard deviation $\sigma=0.30$ (dotted line). Dashed line gives Poisson distribution. Disorder average is over $10^{3}$ configurations.

Fig. 24 left shows the $L$-dependence of the average inverse compressibility, which is well fitted by the power law $\left\langle\Delta_{2}(U)>\propto L^{-\alpha(U)}\right.$, with $\alpha(U)$ going from 2 to 1 when $U$ goes from 0 to 3 approximately. The value $\alpha=2$ is expected without interaction $\left(<\Delta_{2}>=<\Delta>\propto 1 / L^{2}\right.$, with $\langle\Delta\rangle$ single particle mean level spacing). The exponent $\alpha=1$, in this strongly localized regime, can be related to the Coulomb gap in the single particle density of states [57]: According to Koopmans' theorem (see Refs. $[58,59,60]$ for a thorough discussion about the limits 
of validity of the Koopmans' theorem for disordered quantum dots), one assumes that all the other charges are not reorganized by the addition of an extra charge,

$$
\Delta_{2} \approx \epsilon_{N+1}-\epsilon_{N} \propto \frac{1}{L}
$$

due to the Coulomb gap, where $\epsilon_{k}$ is the energy of the $k$-th HF orbital at a fixed number $N$ of particles.

The distribution of inverse compressibilities, for $L=12$ and $W=15$ is shown in Fig. 24 right. At $U=0$, due to Anderson localization, $\Delta_{2}$ distributions are close to the Poisson distribution (deviations from the Poisson distribution at small $s$ values are due to the finite system size). On the contrary, the distribution at $U=3$ shows a Gaussian shape. This can be understood within the Koopmans' theorem, since the HF energies are given by

$$
\epsilon_{k}=\left\langle\psi_{k}\left|H_{1}\right| \psi_{k}\right\rangle+\sum_{\alpha=1}^{N}\left(Q_{\alpha k}^{\alpha k}-Q_{\alpha k}^{k \alpha}\right)
$$

with $H_{1}$ one-body part of the spinless Hamiltonian and $Q_{\alpha \beta}^{\gamma \delta}$ interaction matrix elements given by Eq. (C.3) in appendix C. Due to the small correlations of eigenfunctions in a random potential, one can reasonably invoke the central limit theorem (see Ref. [60] for a more detailed discussion). We point out that a Gaussian-like distribution of inverse compressibilities has been observed in quantum dots experiments in the Coulomb blockade regime $[61,62,63]$. This implies that inverse compressibility fluctuations are dominated by interaction effects instead of single particle fluctuations.

We note that the Gaussian-like shape of the inverse compressibility distribution becomes more asymmetric when the disorder strength is increased. This asymmetry is due to the Coulomb gap and has been discussed in the classical limit $(t=0)$ in Ref. [64].

A simple model for the addition spectra of quantum dots has been recently proposed by B. Shapiro [65]. This model explains the change of the inverse compressibility distribution when $r_{s}$ increases assuming that the system can be decomposed into a stable N-electron Wigner lattice plus a lattice of interstitial sites where the added particle can move and for which a usual single particle description is assumed. This suggests possible relations between the problem of adding an extra particle to a Wigner solid and its melting when $r_{s}$ decreases. 


\section{CONCLUSION}

We have numerically revisited the quantum-classical crossover from the weak coupling Fermi limit towards the strong coupling Wigner limit, using small lattice models. In the continuous limit, the assumed picture [17] is relatively simple: a liquid-solid first order transition for $r_{s} \approx 37$. For a clean lattice model at a filling factor $1 / 9$, our results raise the question of the possible existence of an intermediate liquid-solid phase as first proposed by Andreev and Lifshitz. This may also raise questions about the differences between the continuous limit and lattice models. When the spin degrees of freedom are included, our lattice model is a Hubbard model with additional long range repulsions far from half filling. The physics of such models is not very well known, but at least suspected to be very rich and complex, as discussed in many works since the discovery of high $T_{c}$ superconductors. The role of the disorder is another source of complexity. We have divided this chapter in two main parts, the first where the disorder is weak, the second where the disorder is strong and makes the quantum kinetic effects less relevant. This raises also the question of the difference between the quantum Wigner glass and the classical Coulomb glass. For large disorder, a finite size scaling analysis leads us to conclude that the weak coupling Fermi phase is delimited by a second order quantum phase transition, when $r_{s}$ increases.

Eventually, our first motivation was the experimental discovery of a possible new metal for intermediate values of $r_{s}$. Our numerical studies have not directly addressed the transport properties for intermediate $r_{s}$. One cannot claim that the observed intermediate regime gives a new metal in the thermodynamic limit. Nevertheless, if transport is mainly due to the presence of delocalized defects in a floppy and pinned electron solid for intermediate $r_{s}$, it might be interesting to extend the FLT theory à la Landau proposed in Ref. [24] for a clean system to the case where one has also elastic scattering by impurities. It will tell us if a modified FLT adapted to a very special Fermi system with a non conventional Fermi surface, still gives rise to usual quantum interferences leading to weak localization and eventually to Anderson localization for the conjectured gapless excitations of a floppy solid. This could help to determine whether one has a true new metal for intermediate $r_{s}$ and weak disorder, or only an "apparent" metallic behavior which should disappear at the true $T \rightarrow 0$ limit. This might help to explain why in measurements down to $5 \mathrm{mK}$ in a GaAs hetrostructure [36], the weak localization correction remains less than a few percent of the value predicted for a standard disordered $2 d$ Fermi liquid. 


\section{Appendix: Hartree-Fock approximation}

This is the usual mean field approximation when one has electron-electron interactions, which we shortly review for the case of spinless fermions. The HF ground state (GS) is the Slater Determinant (SD) which minimizes the GS energy expectation value. In the $\mathrm{HF}$ approximation, one reduces the two-body part of the total Hamiltonian to an effective single particle Hamiltonian [66, 67, 68]

$$
U\left(\sum_{i \neq j} \frac{1}{r_{i j}} n_{i}\left\langle n_{j}\right\rangle-\sum_{i \neq j} \frac{1}{r_{i j}} c_{i}^{\dagger} c_{j}\left\langle c_{j}^{\dagger} c_{i}\right\rangle\right),
$$

where $\langle\ldots\rangle$ stands for the expectation value with respect to the HF ground state, which has to be determined self-consistently. The first (Hartree) term describes the interaction of any electron with the charge distribution set up by all the other electrons, while the latter (Fock) term is a nonlocal exchange potential. The Hartree term comes out as an extra on-site disorder potential, while the Fock term introduces extra hopping amplitudes.

The main advantage of the Hartree-Fock approximation is that it reproduces the single-particle density of states $g(E)$ [68], particularly the Coulomb gap near the Fermi energy $E_{F}$ : in the two-dimensional case, $g(E) \propto\left|E-E_{F}\right|$ [57]. The physical argument underlying this relation is that an empty site $j$ and an occupied site $i$ with a difference in energy $\epsilon_{j}-\epsilon_{i}$ smaller than $\delta$ must be at a distance larger than $U / \delta$ as the change $\Delta E_{i j}=\epsilon_{j}-\epsilon_{i}-U / r_{i j}$ of the system energy must be positive when we consider an excitation starting from the ground state. However, the HF approximation gives a Coulomb gap also in the delocalized regime at small disorder (single particle localization length larger than the system size), where complicated many-body effects beyond HF approximation should screen Coulomb interaction.

\section{Appendix: Stoner instability}

Ferromagnetic instabilities come from the interplay between Coulomb repulsion and the Pauli principle. In the Pauli picture, electrons populate the orbital states of a system, such as a quantum dot or a metallic grain, in a sequence of spin up - spin down electrons. The resulting minimum spin state minimizes the kinetic energy: it costs energy to flip a spin since it must be promoted to a higher energy level. On the other hand, the maximum spin state requires a maximally antisymmetric coordinate wavefunction, thus reducing the effect of Coulomb repulsion. This is at the basis of Hund's rule for atoms: electrons occupy orbitals in an open shell so as to maximize their total spin.

Due to the locality of the Pauli principle, ferromagnetic instabilities can be studied within the Hubbard Hamiltonian,

$$
H=-t \sum_{<i, j>\sigma} c_{i \sigma}^{\dagger} c_{j \sigma}+\sum_{i \sigma} v_{i} n_{i \sigma}+U \sum_{i} n_{i \uparrow} n_{i \downarrow} .
$$

In the HF approximation the interaction part of the Hubbard Hamiltonian (B.1) is reduced to

$$
U \sum_{i \sigma} n_{i \sigma}\left\langle n_{i-\sigma}\right\rangle
$$


The HF energies are given by

$$
\epsilon_{\alpha \sigma}=\epsilon_{\alpha}^{0}+U \sum_{i \alpha \beta}\left|\psi_{\alpha}(i)\right|^{2}\left|\psi_{\beta}(i)\right|^{2}\left\langle n_{\beta-\sigma}\right\rangle,
$$

with $\epsilon_{\alpha}^{0}$ one-body eigenenergies and $n_{\beta-\sigma}=d_{\beta-\sigma}^{\dagger} d_{\beta-\sigma}$ occupation numbers for the HF orbitals. The total ground state HF energy reads

$$
E_{0}=\sum_{\alpha \sigma} \epsilon_{\alpha}^{0}\left\langle n_{\alpha \sigma}\right\rangle+U \sum_{i \alpha \beta}\left|\psi_{\alpha}(i)\right|^{2}\left|\psi_{\beta}(i)\right|^{2}\left\langle n_{\alpha \uparrow}\right\rangle\left\langle n_{\beta \downarrow}\right\rangle .
$$

In order to study the stability of the nonmagnetic solution,

$$
\left\langle n_{\alpha \sigma}\right\rangle=\left\{\begin{array}{l}
1 \text { if } \epsilon_{\alpha \sigma}<\epsilon_{F} \\
0 \text { if } \epsilon_{\alpha \sigma}>\epsilon_{F}
\end{array}\right.
$$

with $\epsilon_{F}$ Fermi level, we take a layer (of thickness $\delta \epsilon$ ) of electrons with spin down below the Fermi level to put them in states with spin up [69]. This changes the spin state of $\delta n_{s}=\rho\left(\epsilon_{F}\right) \delta \epsilon$ electrons per unit volume, where $\rho\left(\epsilon_{F}\right)$ is the density of states per volume per spin. The change of the one-body energy density is given by

$$
\delta w_{0}=\rho\left(\epsilon_{F}\right)(\delta \epsilon)^{2} .
$$

In the clean case, the change of the interaction energy (per volume) is

$$
\delta w_{\text {int }}=U\left(\frac{n_{s}}{2}+\rho \delta \epsilon\right)\left(\frac{n_{s}}{2}-\rho \delta \epsilon\right)-\frac{U n_{s}^{2}}{4}=-U \rho^{2}\left(\epsilon_{F}\right)(\delta \epsilon)^{2} .
$$

Therefore the nonmagnetic state becomes unstable when $\delta w_{0}+\delta w_{\text {int }}<0$, that is

$$
U \rho\left(\epsilon_{F}\right)>1
$$

which gives the Stoner criterion for ferromagnetic instability.

In the diffusive regime, the effective interaction strength is enhanced by the presence of disorder, leading to ferromagnetic instabilities already below the Stoner threshold, as pointed out in Ref. [70].

We also note that Stoner criterion predicts a ferromagnetic ground state for the Hubbard model even at finite temperatures in one and in two dimensions, thus violating the Mermin and Wagner's theorem. Therefore, when the Stoner criterion (B.8) is satisfied, we can only conclude that the nonmagnetic ground state is unstable.

The problem of a possible magnetization of the ground state is not only discussed in a dilute $2 \mathrm{DEG}$, but is also central in the studies of mesoscopic quantum dots since their Ohmic resistances are measured as a function of a gate voltage in the Coulomb blockade regime. The possibility of a spontaneous magnetization $S$ of their ground state due to electron-electron interactions has been proposed to explain the observed conductance peak spacing distributions.

\section{Appendix: Configuration interaction method}

Even though the approximations involved in the HF method are uncontrolled, the mean field HF results can be improved using a numerical method $[71,72,56]$ familiar 
in quantum chemistry as the configuration interaction method (CIM) [73]. Once a complete orthonormal basis of HF orbitals has been calculated,

$$
H_{H F}\left(\left|\psi_{1}\right\rangle, \ldots,\left|\psi_{N}\right\rangle\right)\left|\psi_{\alpha}\right\rangle=\epsilon_{\alpha}\left|\psi_{\alpha}\right\rangle
$$

with $\alpha=1,2, \ldots, L^{2}$, it is possible to build up a Slater determinants' basis for the many-body problem which can be truncated to the $N_{H}$ first Slater determinants, ordered by increasing energies. The two-body Hamiltonian can be written as

$$
H_{\mathrm{int}}=\frac{1}{2} \sum_{\alpha, \beta, \gamma, \delta} Q_{\alpha \beta}^{\gamma \delta} d_{\alpha}^{\dagger} d_{\beta}^{\dagger} d_{\delta} d_{\gamma},
$$

with

$$
Q_{\alpha \beta}^{\gamma \delta}=U \sum_{i \neq j} \frac{\psi_{\alpha}(i) \psi_{\beta}(j) \psi_{\gamma}(i) \psi_{\delta}(j)}{r_{i j}}
$$

and $d_{\alpha}^{\dagger}=\sum_{j} \psi_{\alpha}(j) c_{j}^{\dagger}$. One gets the residual interaction subtracting Eq. A.1 from Eq. C.2.

\section{Acknowledgments}

The authors wish to acknowledge the participation of D. Shepelyansky in one of the reviewed works (Ref. [56]) and to thank B. Spivak for discussions about the work of Andreev and Lifshitz.

Present addresses:

G. Benenti: Università degli Studi dell'Insubria and Istituto Nazionale per la Fisica della Materia, via Valleggio 11, 22100 Como, Italy.

G. Katomeris: Department of Physics, University of Ioannina, 45 110, Greece.

X. Waintal: Laboratory of Atomic and Solid State Physics 530 Clark Hall, Cornell University, Ithaca, NY 14853-2501, USA.

\section{References}

[1] E. P. Wigner, Trans. of the Faraday Soc. 34, 678 (1938). See also Phys. Rev. 46, $1002(1934)$

[2] L. D. Landau, JETP 30, 1058 (1956).

[3] B. L. Altshuler and A. G. Aronov, in Electron-Electron Interactions in Disordered Systems, edited by A. L. Efros and M. Pollak (North Holand, Amsterdam, 1985).

[4] E. Abrahams, S. V. Kravchenko and M. P. Sarachik, cond-mat/0006055, to be published in Rev. Mod. Phys. 73, 251 (2001) and Refs. therein.

[5] M. Y. Simmons, A. R. Hamilton, M. Pepper, E. H. Linfeld, P. D. Rose and D. A. Ritchie, Phys. Rev. Lett. 84, 2489 (2000).

[6] Y. Yaish, O. Prus, E. Buchstab, S. Shapira, G. Ben Joseph, U. Sivan and A. D. Stern, Phys. Rev. Lett. 85, 4954 (2000).

[7] V. Senz, T. Ihn, T. Heinzel, K. Ensslin, G. Dehlinger, D. Grutzmacher and U. Gennser, Phys. Rev. Lett. 85, 4357 (2000).

[8] B. L. Altshuler and D. L. Maslov, Phys. Rev. Lett. 82, 145 (1999).

[9] S. S. Safonov, S. H. Roshko, A. K. Savchenko, A. G. Pogosov, and Z. D. Kvon, Phys. Rev. Lett. 86, 272 (2001).

[10] Y. Meir, Phys. Rev. Lett. 83, 3506 (1999). 
[11] A. P. Mills, A. P. Ramirez, L. N. Pfeiffer and K. W. West, Phys. Rev. Lett. 83, 2805 (1999).

[12] J. H. Schön, S. Berg, Ch. Kloc and B. Batllog, Science 287, 1022 (2000).

[13] Lynn Bonsall and A. A. Maradudin, Phys. Rev. B 15 (1977) 1959.

[14] D. Pines The many body problem, W. A. Benjamin, Inc. New York (1961).

[15] W. J. Carr, Phys. Rev. 122, 1437 (1961).

[16] D. Ceperley, Phys. Rev. B 18, 3126 (1978).

[17] B. Tanatar and D. Ceperley, Phys. Rev. B 39, 5005 (1989).

[18] D. Varsano, S. Moroni and G. Senatore, Europhys. Lett. 53, 348 (2000).

[19] Ladir Cândido, Philip Phillips and D. M. Ceperley, Phys. Rev. Lett. 86, 492 (2001).

[20] B. Bernu, L. Cândido and D. M. Ceperley, cond-mat/9908062.

[21] M. Roger, Phys. Rev. B 30, 6432 (1984).

[22] S. T. Chui and B. Tanatar, Phys. Rev. Lett. 74, 458 (1989).

[23] A. F. Andreev and I. M. Lifshitz, JETP 29 (1969) 1107.

[24] I. E. Dzyaloshinskii, P. S. Kondatenko and V. S. Levchenko, JETP 35 (1972) 823 and 1213.

[25] J. H. Schön, Ch. Kloc and B. Batllog, Letters to Nature 406, 702 (2000).

[26] J. H. Schön, Ch. Kloc and B. Batllog, Science 288, 2338 (2000).

[27] B. Batllog, colloqium given in Orsay (october 2000).

[28] E. Y. Andrei, G. Deville, D. C. Glattli, F. I. B. Williams, E. Paris and B. Etienne, Phys. Rev. Lett. 60, 2765 (1988).

[29] A. A. Shashkin, S. V. Kravchenko and T. M. Klapwijk, cond-mat/0009180.

[30] S. V. Kravchenko and T. M. Klapwijk, Phys. Rev. Lett. 84, 2909 (2000).

[31] S. A. Vitkalov, H. Zheng, K. M. Mertes, M. P. Sarachik and T. M. Klapwijk, Phys. Rev. Lett. 85, 2164 (2000).

[32] S. A. Vitkalov, H. Zheng, K. M. Mertes, M. P. Sarachik and T. M. Klapwijk, cond-mat/0009454.

[33] J. Yoon, C. C. Li, D. Shahar, D. C. Tsui and M. Shayegan, Phys. Rev. Lett. 82, 1744 (1999).

[34] A. M. Finkelshtein, Sov. Phys. JETP 57, 98 (1983).

[35] A. R. Hamilton, M. Y. Simmons, M. Pepper, E. H. Linfield, P. D. Rose and D. A. Ritchie, Phys. Rev. Lett. 82, 1542 (1999).

[36] A. P. Mills, Jr., A. P. Ramirez, X. P. A. Gao, L. N. Pfeiffer, K. W. West and S. H. Simon, cond-mat/0101020.

[37] P. Mohanty, E. M. Q. Jariwala and R. A. Webb, Phys. Rev. Lett. 77, 3366 (1997).

[38] C. Dultz and H. W. Jiang, Phys. Rev. Lett. 84, 4689 (2000).

[39] S. Ilani, A. Yacoby, D. Mahalu and Hadas Shtrikman, Phys. Rev. Lett. 84, 3133 (2000).

[40] S. Ilani, A. Yacoby, D. Mahalu and Hadas Shtrikman, Science 292, 1354 (2001).

[41] M. Reznikov, conference talk given in Rencontres de Moriond, Les Arcs, 2001.

[42] G. Benenti, X. Waintal and J.-L. Pichard, Phys. Rev. Lett. 83, 1826 (1999).

[43] R. Berkovits and Y. Avishai, Phys. Rev. B 57, R15076 (1998).

[44] F. Selva and D. Weinmann, Eur. Phys. J. B 18, 137 (2000).

[45] G. Benenti, X. Waintal and J.-L. Pichard, Europhys. Lett. 51, 89 (2000).

[46] G. Katomeris and J.-L. Pichard, cond-mat/0012213. 
[47] F. Selva and J.-L. Pichard, cond-mat/0012015.

[48] R. C. Ashoori, Nature 379, 413 (1996).

[49] D. H. Dubin and T. M. O’Neil, Rev. Mod. Phys. 71, 87 (1999).

[50] N. B. Zhitenev, M. Brodsky, R. C. Ashoori, L. N. Pfeiffer and K. W. West, Science 285, 715 (1999).

[51] C. Yannouleas and U. Landman, Phys. Rev. Lett. 85, 1726 (2000).

[52] A. V. Filinov, M. Bonitz and Yu. E. Lozovik, Phys. Rev. Lett. 86, 3851 (2001).

[53] X. Waintal, G. Benenti, and J.-L. Pichard, Europhys. Lett. 49, 466 (2000).

[54] J.-L. Pichard and G. Sarma, J. Phys. C 14, L127 and L617 (1981).

[55] A. MacKinnon and B. Kramer, Phys. Rev. Lett. 47, 1546 (1981); B. Kramer and A. MacKinnon, Rep. Prog. Phys. 56, 1469 (1993).

[56] G. Benenti, X. Waintal, J.-L. Pichard, and D.L. Shepelyansky, Eur. Phys. J B 17, 515 (2000).

[57] A.L. Efros and B.I. Shklovskii, J. Phys. C 8, L49 (1975); M. Pollak, Phil. Mag. B 65, 657 (1992); see also Electron-Electron Interactions in Disordered Systems, edited by A.L. Efros and M. Pollak, (North-Holland, Amsterdam, 1985).

[58] S. Levit and D. Orgad, Phys. Rev. B 60, 5549 (1999).

[59] P.N. Walker, G. Montambaux, and Y. Gefen, Phys. Rev. B 60, 2541 (1999).

[60] A. Cohen, K. Richter, and R. Berkovits, Phys. Rev. B 60, 2536 (1999).

[61] U. Sivan, R. Berkovits, Y. Aloni, O. Prus, A. Auerbach, and G. Ben-Yoseph, Phys. Rev. Lett. 77, 1123 (1996).

[62] S.R. Patel, S.M. Cronenwett, D.R. Stewart, A.G. Huibers, C.M. Marcus, C.I. Duruöz, J.S. Harris, Jr., K. Campman, and A.C. Gossard, Phys. Rev. Lett. 80, 4522 (1998).

[63] F. Simmel, D. Abusch-Magder, D.A. Wharam, M.A. Kastner, and J.P. Kotthaus, Phys. Rev. B 59, R10441 (1999).

[64] A.A. Koulakov, F.G. Pikus, and B.I. Shklovskii, Phys. Rev. B 55, 9223 (1997).

[65] B. Shapiro, cond-mat/0008366, to appear in Phil. Mag. 82, No 3.

[66] H. Kato and D. Yoshioka, Phys. Rev. B 50, 4943 (1994).

[67] G. Bouzerar and D. Poilblanc, J. Phys. I France 7, 877 (1997).

[68] F. Epperlein, M. Schreiber, and T. Vojta, Phys. Rev. B 56, 5890 (1997); Phys. Status Solidi (b) 205, 233 (1998).

[69] See, e.g., A. Blandin, in Magnetism - Selected topics, edited by S. Foner (Gordon and Breach, New York, 1976).

[70] A.V. Andreev and A. Kamenev, Phys. Rev. Lett. 81, 3199 (1998).

[71] M. Eto amd H. Kamimura, Phys. Rev. Lett. 61, 2790 (1988).

[72] T. Vojta, F. Epperlein, and M. Schreiber, Phys. Rev. Lett. 81, 4212 (1998).

[73] P. Fulde, Electron Correlations in Molecules and Solids, (Springer, Berlin, 1995). 